\title{
Enzymatic control over reactive intermediates enables direct oxidation of alkenes to carbonyls by a P450 iron-oxo species
}

\author{
Jordi Soler, ${ }^{a, \ddagger}$ Sebastian Gergel, ${ }^{b, \ddagger}$ Stephan C. Hammer, ${ }^{b, *}$ Marc Garcia-Borràs ${ }^{a, *}$ \\ a Institut de Química Computacional i Catàlisi (IQCC) and Departament de Química, Universitat de Girona, \\ Carrer Maria Aurèlia Capmany 69, Girona 17003, Catalonia, Spain \\ ${ }^{\text {b }}$ Chair of Organic Chemistry and Biocatalysis, Faculty of Chemistry, Bielefeld University, Universitätsstraße 25, \\ 33615 Bielefeld, Germany \\ (†: these authors contributed equally)
}

\begin{abstract}
The oxidation of alkenes by high-valent iron-oxo active species normally leads to the formation of the corresponding epoxides. Rarely, carbonyl compounds are observed as a side product of the reaction. However, the selective formation of carbonyl compounds from alkenes using iron-oxo species and small molecule catalysts has not yet been achieved. Recently, a new P450-based enzyme (aMOx) has been engineered through laboratory directed evolution to directly oxidize styrenes to their corresponding aldehydes. This transformation uses an enzymatic iron-oxo species as catalytic oxidant and generates aldehydes with high activity and selectivity while suppressing epoxidation.
\end{abstract}

Here, we combine extensive computational modelling together with experimental mechanistic investigations to study the reaction mechanism and unravel the molecular basis behind the selectivity achieved by the laboratory evolved aMOx enzyme. We found that alkene epoxidation and carbonyl formation pathways diverge from a common covalent radical intermediate generated after the first $\mathrm{C}-\mathrm{O}$ bond formation. Although both pathways are accessible and very similar in energy, intrinsic dynamic effects determine the strong preference for epoxidation. We discovered that aMOx overrides these intrinsic dynamic preferences by controlling the accessible conformations of the covalent radical intermediate. This disfavors epoxidation and facilitates the formation of a key carbocation intermediate that generates the aldehyde product through a fast 1,2-hydride migration. Computations predicted that the hydride migration is stereoselective due to the conformational control over the intermediate species when formed in the enzyme active site. These predictions were corroborated by experiments using deuterated styrene substrates, which proved that the hydride migration is cis- and enantioselective.

Our results demonstrate that directed evolution tailored a highly specific active site that imposes strong steric control over key fleeting biocatalytic intermediates, which is essential for accessing the carbonyl forming pathway and preventing competing epoxidation. 


\section{Introduction}

The aerobic oxidation of alkenes to the corresponding carbonyl compounds is an important but often challenging transformation in organic chemistry. ${ }^{1-3}$ The value of this oxidation reaction results from the easy accessibility of alkenes from petroleum, renewable resources or well-established synthetic methods such as carbonyl olefination and olefin metathesis. In addition, aldehydes and ketones are very relevant functional groups in valuable molecules as well as crucial intermediates in synthesis. Alkene to carbonyl oxidation is established by the palladium(II)-catalyzed Wacker-Tsuji oxidation, yet, this reaction is in part limited to oxidize ethylene to acetaldehyde and 1-alkenes to methyl ketones. ${ }^{[1,2]}$ Although significant efforts have been made to extend Wacker-type oxidations to yield other types of carbonyl compounds, ${ }^{1-3}$ efficient and selective aerobic alkene to carbonyl oxidations are rare and sought after.

In chemistry and biology, high-valent metal-oxo complexes are important catalytic species for oxidation chemistry. ${ }^{4-7}$ However, these catalytic oxidants are typically used to epoxidize alkenes. Established catalysts include Jacobsen's manganese(III)salen complexes ${ }^{8}$, synthetic metalloporphyrins ${ }^{9}$, biomimetic non-heme iron complexes ${ }^{10-12}$ and enzymes such as hemedependent cytochrome P450 monooxygenase ${ }^{13}$ and peroxygenases, ${ }^{14}$ or non-heme $\alpha$ ketoglutarate-dependent dioxygenases. ${ }^{15}$ The exact mechanism of metal-oxo-mediated alkene epoxidation has long been controversial. ${ }^{7,16,17} \mathrm{~A}$ currently widely accepted view is that epoxide formation proceeds via an almost concerted reaction mechanism, potentially through an extremely short lived radical intermediate (Scheme 1). Support for a significant concerted nature of the oxo transfer, or a two-step mechanism in which the second step occurs very fast, comes from the retention of stereochemistry in epoxidation of cis-alkenes. ${ }^{8,9,13,14}$ Next to epoxidation, experiments and theory suggest that high-valent metal-oxo species can produce carbonyl compounds in a direct oxo transfer reaction. ${ }^{16,18,19}$ It is believed that metal-oxomediated alkene to carbonyl oxidation proceeds from the radical intermediate via an electron/hydride transfer process including the formation of a carbocation species (Scheme 1). ${ }^{19,20}$ This proposed electron transfer process is related to the carbocation formation in metal-oxo-mediated oxidative rearrangements, for example in pentalenolactone biosynthesis, catalyzed by a P450 enzyme that includes an intramolecular electron transfer. $^{21,22}$ Although direct alkene to carbonyl oxidation is theoretically accessible through oxo transfer chemistry, carbonyl products are only observed as minor by-products, if at all. ${ }^{16,20}$ High-valent metal-oxo species have never been exploited for carbonyl synthesis due to the lack of catalysts that suppress the strongly favored epoxide formation. ${ }^{23}$ The origin of high chemoselectivity that favors epoxidation over carbonyl formation is not completely understood. Recently, however, directed evolution has been harnessed to generate a biocatalyst that 
outcompetes epoxidation. A laboratory evolved iron-heme P450 enzyme directly oxidizes alkenes to carbonyls with high activity and selectivity (up to $94 \%$ carbonyl selectivity and up to 4500 total turnover numbers, TTN). ${ }^{23}$ The enzyme was evolved from a P450 monooxygenase that use a high-valent metal-oxo species (termed compound I, Cpd I) as catalytic oxidant. This is the first catalyst to use metal-oxo species for selective alkene to carbonyl oxidation and thus a good opportunity to study the origin of chemoselectivity in this reaction.

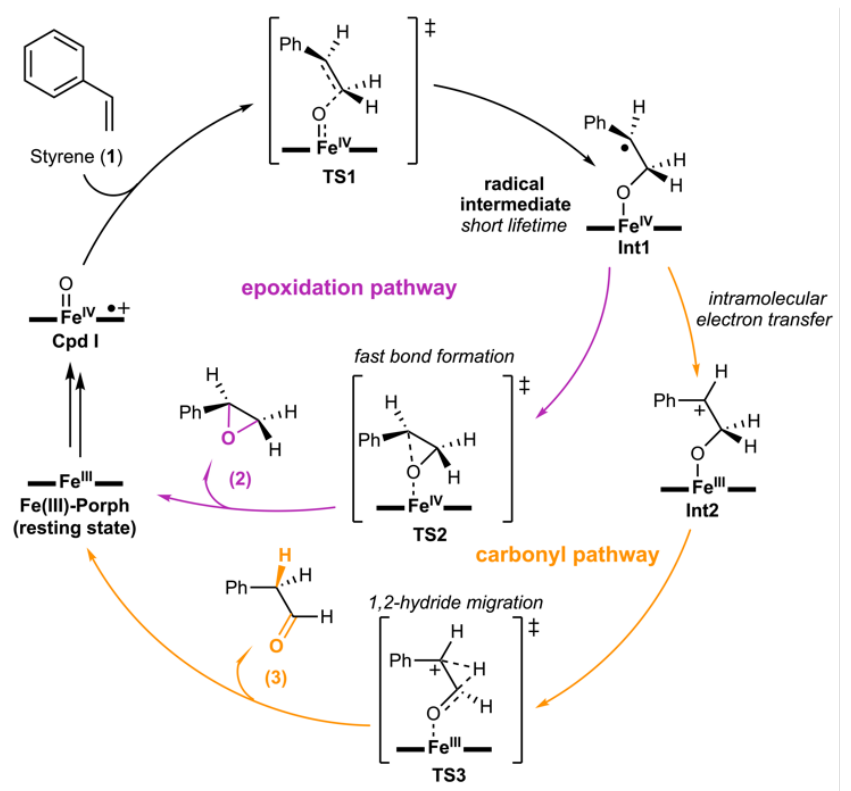

Scheme 1. Fe-oxo-mediated alkene oxidation. Fe-oxo-mediated alkene oxidation generally leads to the corresponding epoxide product. Proposed catalytic cycles for P450-catalyzed alkene epoxidation (epoxide pathway, purple) and anti-Markovnikov oxidation (carbonyl pathway, orange) with styrene (1) as model substrate starting with the formation of an iron-oxo complex, termed compound I (Cpd I). The first C-O bond formation (TS1) leads to a short-lived radical intermediate (Int-1) that directly converts to the epoxide product (2) by a very fast second C-O bond forming step (TS2). These two C-O bond forming steps proceed most often in a stereospecific manner and might occur stepwise (without epimerization when the shallow reactive radical intermediate is formed) or in a concerted fashion. The alternative stepwise anti-Markovnikov oxidation (carbonyl pathway) is proposed to occur via an intramolecular electron transfer, yielding a highly reactive carbocation intermediate (Int2). Subsequent 1,2-hydride migration (TS3) produces the carbonyl product, aldehyde 3.

As we will describe below, iron-oxo-mediated alkene oxidation can involve a short-lived radical intermediate that is generated with large excess of kinetic energy (Scheme 2). This is not unusual and in fact common to any intermediate or product derived from a high in energy transition state (TS). ${ }^{24}$ This excess energy originates from potential energy that is in part transformed into vibrational energy right after the intermediate or product formation (Scheme 2A). Because energy redistribution via intramolecular and intermolecular processes is extremely fast in solution, ${ }^{25}$ excess vibrational energy does typically not affect subsequent reactions of an intermediate. Consequently, statistical thermodynamics treatments, such as transition state theory, can be successfully applied to rationalize and predict experimental observations. However, this is not the case if the lifetime of a reactive intermediate is very 
short and reaches the femto- to picosecond timescale. At this timescale, the reactivity of the intermediate can compete with its excess energy redistribution and thermal equilibration. ${ }^{24}$ For reactive intermediates with very short lifetimes, non-statistic distribution of energy can affect their dynamic behavior and thus control the reactivity of these species (Scheme 2B). Changes in reactivity can for example take place via selective vibrational activation of one part of a molecule or by specific vibrations with a displacement (momentum) that correlates with the geometry of a subsequent transition state and its displacement vector corresponding to the imaginary frequency (dynamic match). The reactivity of energetically non-equilibrated intermediates (kinetically activated, "hot" intermediates) has received significant attention recently. ${ }^{24,26}$ It has been shown that dynamic effects and the amount of excess energy and how it is distributed can control the reactivity of intermediates in solution, ${ }^{27,28}$ in organometallic reactions ${ }^{29,30}$ and in enzymes. ${ }^{31-33}$

Here we show that the high chemoselectivity in metal-oxo mediated alkene oxidation by iron porphyrin-type catalysts is a consequence of the radical's dynamic behavior in its kinetically (vibrational) activated state. We further reveal how an evolved heme-dependent enzyme overwrites this dynamically-controlled process to generate carbonyls instead of epoxides in the oxidation of styrene. Directed evolution of aMOx not only steered oxidation activity towards acceptance of a non-natural substrate, but enabled control over the accessible conformations of the reactive intermediates by preorganizing them to favor stereoselective carbonyl formation while disfavoring epoxidation. This conformational control is achieved by confinement $^{34}$ in the active site of the enzyme. Experiments with isotopically labelled substrates support the computational findings and proposed reaction mechanism.
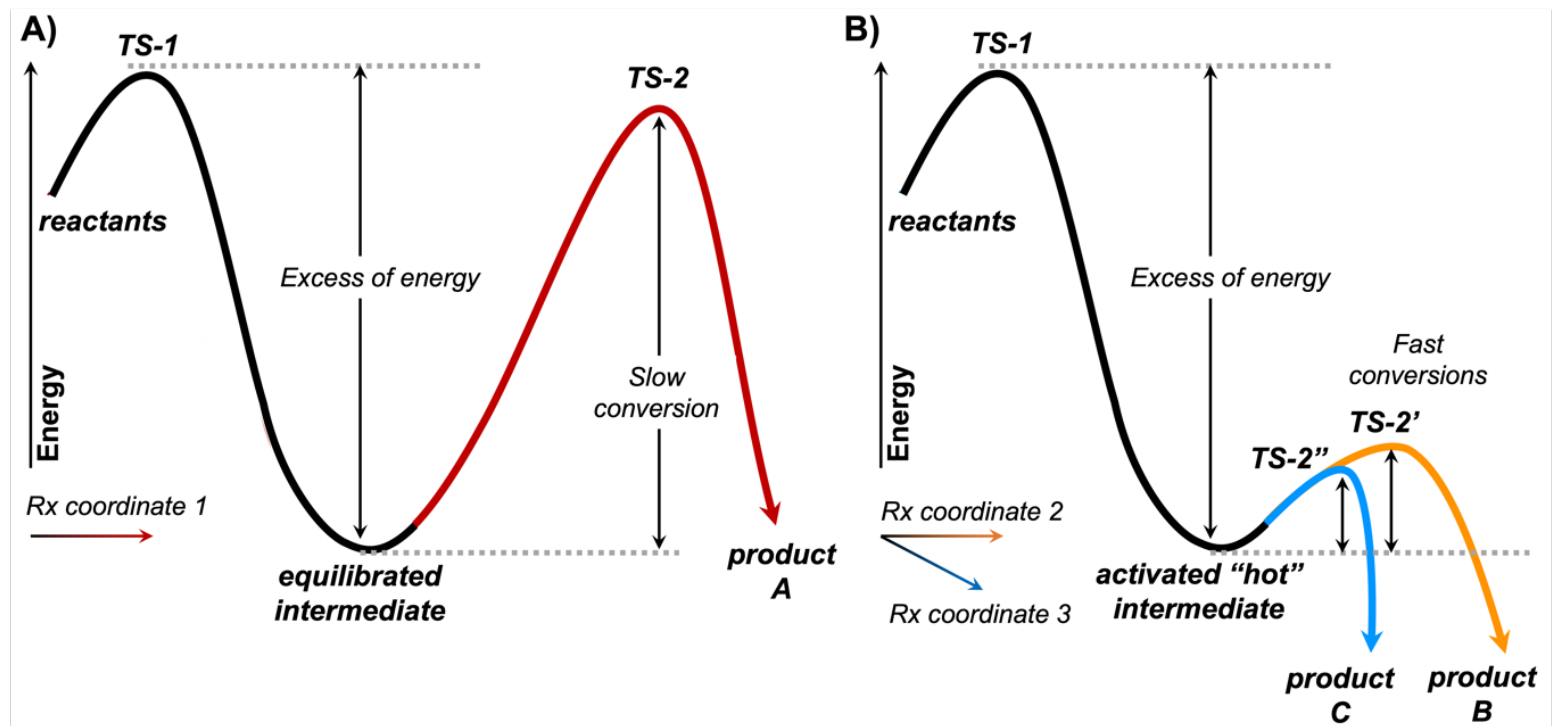

Scheme 2. Activated "hot" intermediates. Kinetically activated ("hot") intermediates are formed with an excess energy that depends on the kinetic energy acquired during the formation of the intermediate (first transition state TS-1). A) A high energy second transition state, TS-2, implies a slow conversion of the intermediate. It allows thermal equilibration of the intermediate, loss of excess energy to the surrounding solvent molecules, and 
intramolecular vibrational energy redistribution (IVR). Transition state theory can be successfully applied to predict reaction rates, intermediate lifetimes and product selectivities. B) A low energy second transition state, TS-2' or TS-2", implies a fast conversion of the intermediate. At short timescales, the reactivity of the intermediate can compete with its excess energy redistribution and thermal equilibration. Consequently, it might be possible that exists a strong coupling of the kinetic energy acquired by the intermediate during its formation with modes that promote its subsequent reaction. In this scenario, transition state theory fails and experimental observables (as selectivities) do not agree with statistical predictions due to dynamic effects. Dynamic behavior of the activated "hot" intermediate could favor the formation of product B through TS-2' (non-intrinsic reaction coordinate, non-IRC pathway) over product C through the lower in energy TS-2"' (lowest in energy intrinsic reaction coordinate, IRC pathway). 


\section{Results and Discussion}

\section{Exploration of the intrinsic oxidation reaction mechanism using DFT calculations on an enzyme-free computational truncated model.}

To understand the origins of chemoselectivity in iron-oxo-mediated alkene oxidation by iron porphyrin-type catalysts, we first studied the intrinsic reaction mechanism using DFT calculations and a simple truncated model (Figure 2, see SI for computational details). This simplified model includes the high valent iron-oxo species coordinated to the porphyrin pyrrole core and a methanethiolate moiety that mimics the axial cysteine ligand in compound I (Cpd I). We used styrene as model alkene substrate because this alkene is typically used in epoxidation reactions and it was also the substrate for the engineered aMOx enzyme that performs chemoselective carbonyl formation. The energetically accessible spin states (doublet, $\mathrm{d}$; and quartet, $\mathrm{q}$ ) of the catalytic iron species have been considered and are discussed if necessary (see SI for details). ${ }^{7,35}$

As styrenes are generally epoxidized by high-valent iron-oxo species, ${ }^{8-14}$ one might expect to find significantly lower energy barriers for epoxidation than for the competing carbonyl pathway. This would be in line with transition state theory and relate product selectivities to differences in activation barriers. However, the calculated reaction mechanism for iron-oxo-mediated styrene oxidation revealed that the carbonyl pathway is not only energetically accessible, but even slightly preferred over epoxidation (Fig. 2A, and Fig. S1). Calculations describe that the formation of the first $\mathrm{C}-\mathrm{O}$ bond between the terminal carbon atom of the styrene substrate and the oxygen of $\mathrm{Cpd} \mathrm{I}$ is the shared rate limiting step for both epoxidation and carbonyl pathways (TS1, $\Delta \mathrm{G}^{\ddagger}=10.9 \mathrm{kcal} \cdot \mathrm{mol}^{-1}$ and $11.0 \mathrm{kcal} \cdot \mathrm{mol}^{-1}$ in the quartet and doublet states, respectively). Different conformers evaluated for TS1 revealed no significant energy differences (see Fig. 2B). Intrinsic reaction coordinate (IRC) calculations (see SI), describe that TS1 in the doublet electronic state directly leads to the epoxide product via formation of the second $\mathrm{C}-\mathrm{O}$ bond in an asynchronous but concerted manner, while quartet TS1 forms a covalent radical intermediate (Int1). The generated radical intermediate can either lead to the epoxide product via a low in energy transition state (TS2, $\Delta \mathrm{G}^{\ddagger}=2.7$ $\mathrm{kcal} \cdot \mathrm{mol}^{-1}$ ), or to the carbonyl product in an almost barrierless pathway. The latter involves the formation of a covalent carbocation intermediate (Int2) that is $\Delta \Delta \mathrm{G}=0.8 \mathrm{kcal} \cdot \mathrm{mol}^{-1}$ higher in energy (quartet) and $-3.8 \mathrm{kcal} \cdot \mathrm{mol}^{-1}$ more stable (doublet) with respect to the radical intermediate (Fig. 2C and Fig. S1). The formation of this key carbocation intermediate requires a conformational change, the rotation of the phenyl ring of the substrate, that enables stabilization of the benzylic carbocation via resonance with the aromatic ring as well as hyperconjugation with the former vinylic $\alpha-\mathrm{C}-\mathrm{H}$ bonds (Fig. 2C, Fig. S1). Within this 
conformation, the lone pairs of the oxygen atom ( $2 p$ orbitals) can stabilize the empty $p$ orbital of the carbocation through a $n \rightarrow p$ interaction. Additionally, a $\mathrm{C}-\mathrm{H} \cdots \mathrm{O}$ interaction can also be established that stabilizes the positive charge partially delocalized on the aromatic ring by resonance. In this optimal conformation, the $\alpha$-hydrogen is well aligned with the empty $p$ orbital of the benzylic carbocation for an effective 1,2-migration. The carbonyl product 3 can be generated in either spin state by barrierless hydride migration (TS3) from carbocation Int2.

Calculations further show that the proposed intramolecular electron transfer of the radical intermediate Int1 to generate the carbocation Int2 is feasible. It is found to involve an inversion of the frontier molecular orbitals (FMO), where the electron from the $\alpha$-HOMO in the radical intermediate moves to the energetically accessible $\alpha-\mathrm{LUMO}+1$ (quartet electronic state) or $\beta$ LUMO (doublet, see Fig. S2). Once formed, the carbocation intermediate Int2 in the quartet electronic state can access the lower in energy doublet Int2 through a minimum energy crossing point (MECP) ${ }^{36}$ that is energetically and geometrically very similar to the quartet Int2 optimized structure (Fig. S3). Finally, DFT calculations also showed that the presence of a precisely positioned water molecule hydrogen-bonding with the $\mathrm{O}$-atom of the intermediate favors the relative stabilization of the carbocation with respect to the radical intermediate (Fig. S4).

DFT calculations described a flat region of the potential energy surface (PES), with the carbonyl formation being slightly energetically favored when diverging from quartet TS1. The lifetime of the radical intermediate predicted by transition state theory for epoxidation pathway (TS2, $\left.\Delta \mathrm{G}^{\ddagger}=2.7 \mathrm{kcal} \cdot \mathrm{mol}^{-1}\right)$ is estimated to be $t_{1 / 2} \approx 1.06 \cdot 10^{-11} \mathrm{~s}(10600 \mathrm{fs})$. Considering a first order reaction, a rate constant of $K=6.52 \cdot 10^{10} \mathrm{~s}^{-1}$ can be derived from the Eyring equation. This is more than one order of magnitude slower than the predicted rate constant for carbonyl formation $\left(K=2.67 \cdot 10^{12} \mathrm{~s}^{-1}\right)$ via TS3 from Int1 (quartet electronic state, $\Delta \mathrm{G}^{\ddagger}=0.5 \mathrm{kcal} \cdot \mathrm{mol}^{-1}$ ). According to transition state theory such reactions should preferentially lead to carbonyl products instead of the experimentally observed epoxides. This reveals that thermostatistical methods such as transition state theory cannot completely explain why metal-oxo mediated iron porphyrin- or P450-catalyzed alkene oxidations favor epoxidation while carbonyl formation is rarely observed as side product. $^{18,20}$

We hypothesized that non-statistical effects and the dynamic behavior of the radical intermediate could determine the chemoselectivity in this oxo transfer reaction. This idea is supported by the very short lifetime predicted for this radical (in the femtosecond timescale) which can thus compete with redistribution of excess of kinetic energy. In this particular case, the radical intermediate is generated from TS1 with a high amount of kinetic energy $(\Delta \Delta \mathrm{G}=$ $22.1 \mathrm{kcal} \mathrm{mol}^{-1}$, see Fig. 2A), rendering it a kinetically activated ("hot") intermediate (Scheme 2). Analysis of the momentum vectors corresponding to the negative eigenvalues of 
TS1 and TS2 already indicate a strong coupling and "dynamic match" between both transitions states (Fig. 3A), suggesting that kinetic activation and resulting non-statistic dynamic behavior of the radical intermediate could strongly favor three membered ring formation.

(A)

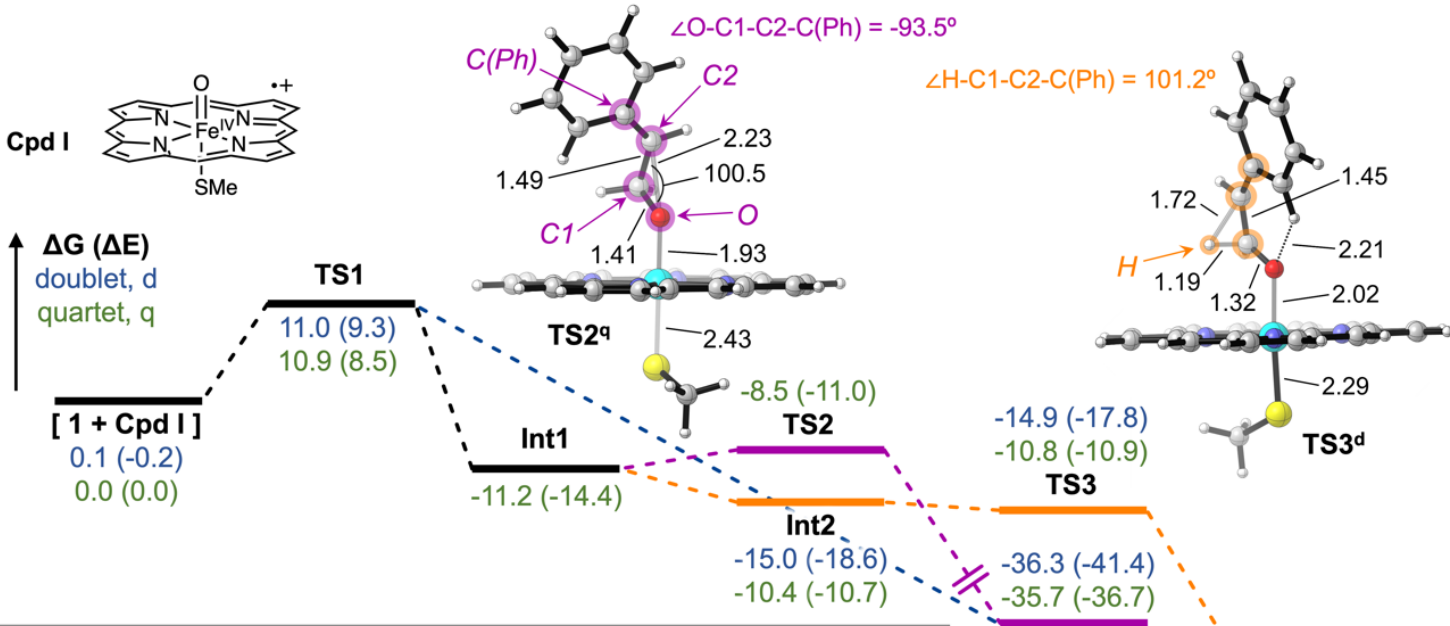

(B)

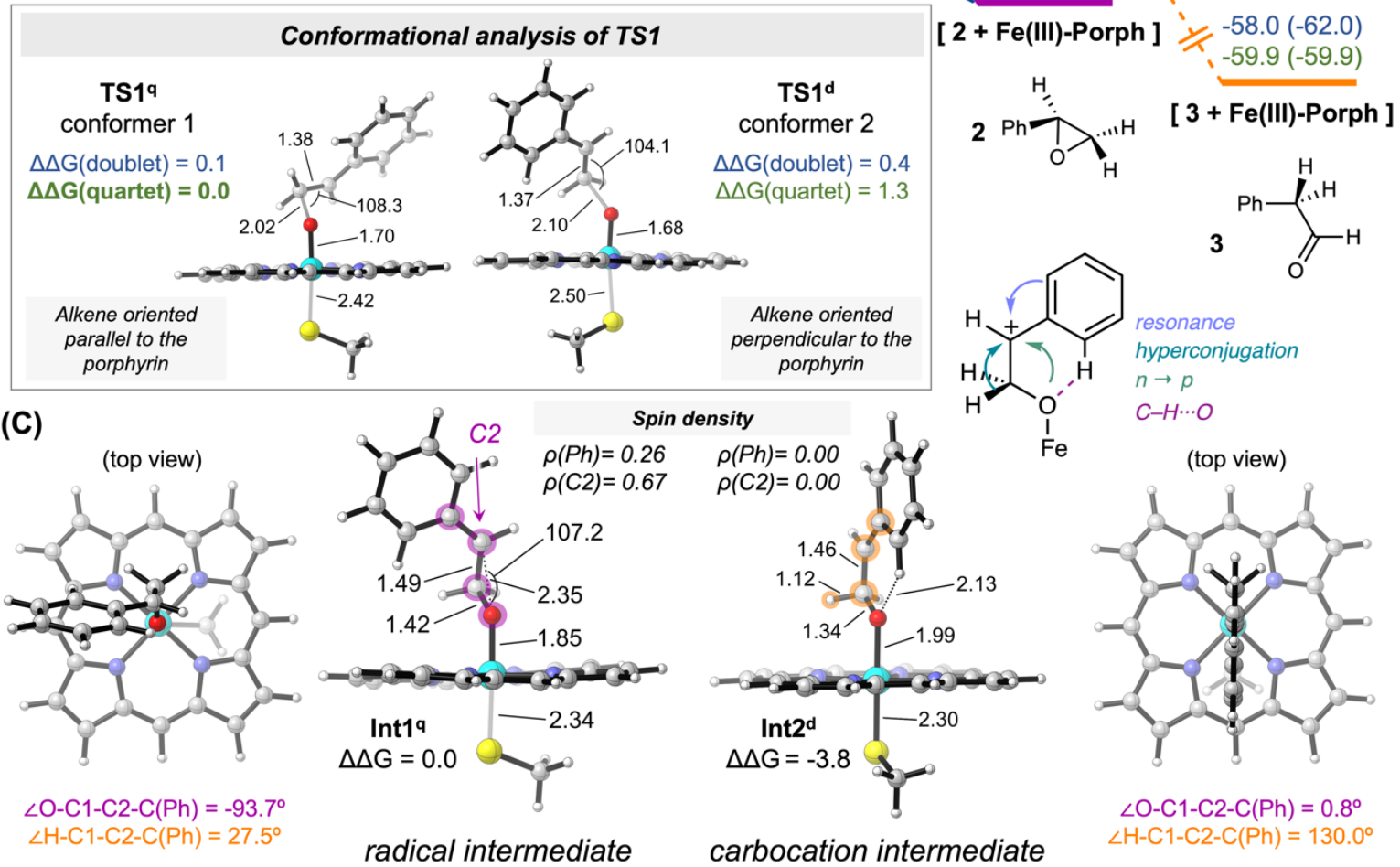

Figure 2. Enzyme-free model DFT calculations. (A) DFT-calculated competing reaction pathways for styrene (1) epoxidation (purple pathway) and carbonyl formation (orange pathway) catalyzed by Fe-oxo heme enzyme-free computational model. Relative Gibbs free energies $(\Delta G)$ and electronic energies $(\Delta E$, in parenthesis) are reported, in orange for the doublet electronic state (d) and in green for the quartet electronic state $(q)$. Lowest in energy DFT optimized key transition states geometries are shown. See Fig. S1 for additional details on the intrinsic mechanisms. (B) Conformational analysis of TS1. (C) Lowest in energy DFT optimized structures of key covalent radical and carbocation intermediates (Int1 and Int2, respectively). Energies, distances, and angles are given in $\mathrm{kcal} \cdot \mathrm{mol}^{-1}$, angstroms $(\AA)$, and degrees $\left(^{\circ}\right)$, respectively.

\section{Quasiclassical direct dynamics trajectory simulations.}


To investigate the influence of non-equally distributed excess of kinetic energy on the chemoselectivity of the reaction (i.e. nonstatistical effects), we used direct quasiclassical trajectory (QCT) calculations to study the reaction dynamics. ${ }^{24,30,37} \mathrm{QCT}$ allows to explicitly consider the atomic motions during a chemical reaction, which could describe the formation of nonstatistical (activated, "hot") intermediates without vibrational energy redistribution, and the possible existence of nonintrinsic reaction pathways (non-IRC). Non-IRC pathways can lead to products that do not correspond to those expected from the lowest in energy pathway (i.e. intrinsic reaction coordinate, IRC). QCT calculations have been previously employed to comprehend selectivities in competing reactions that bifurcate after a shared transition state, to characterize the formation of shallow entropic intermediates along reaction pathways, or to study the dynamic behavior of reactive intermeditates. ${ }^{24,30,37-42}$

Using our computational truncated model, quasiclassical trajectory simulations were carried out (Fig. 3). Trajectories were propagated forwards and backwards for $600 \mathrm{fs}$ (300 fs in each direction) starting from optimized TS1 structures for each energetically accessible spin state (20 trajectories for each electronic state, total of 40 trajectories. See SI for computational details). None of the 40 trajectories accessed the carbonyl product or the carbocation intermediate (Fig. S5). Instead, 7 resulted in non-reactive recrossing events (5 doublet +2 quartet), 7 lead to the formation of the radical intermediate (quartet) and 26 trajectories (15 doublet +11 quartet) generated the epoxide product (see Fig. S5). 
(A)

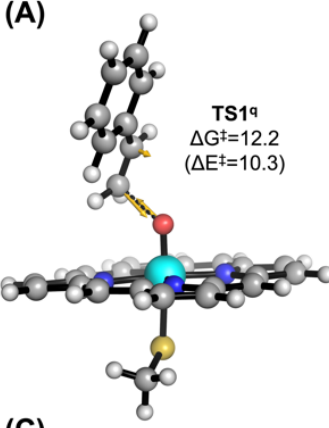

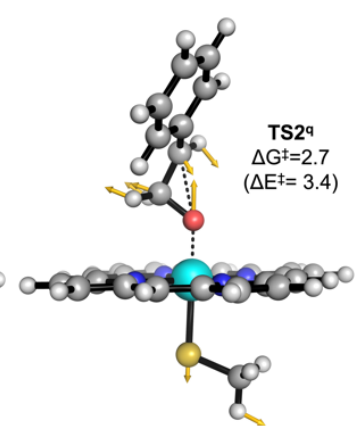

(B)
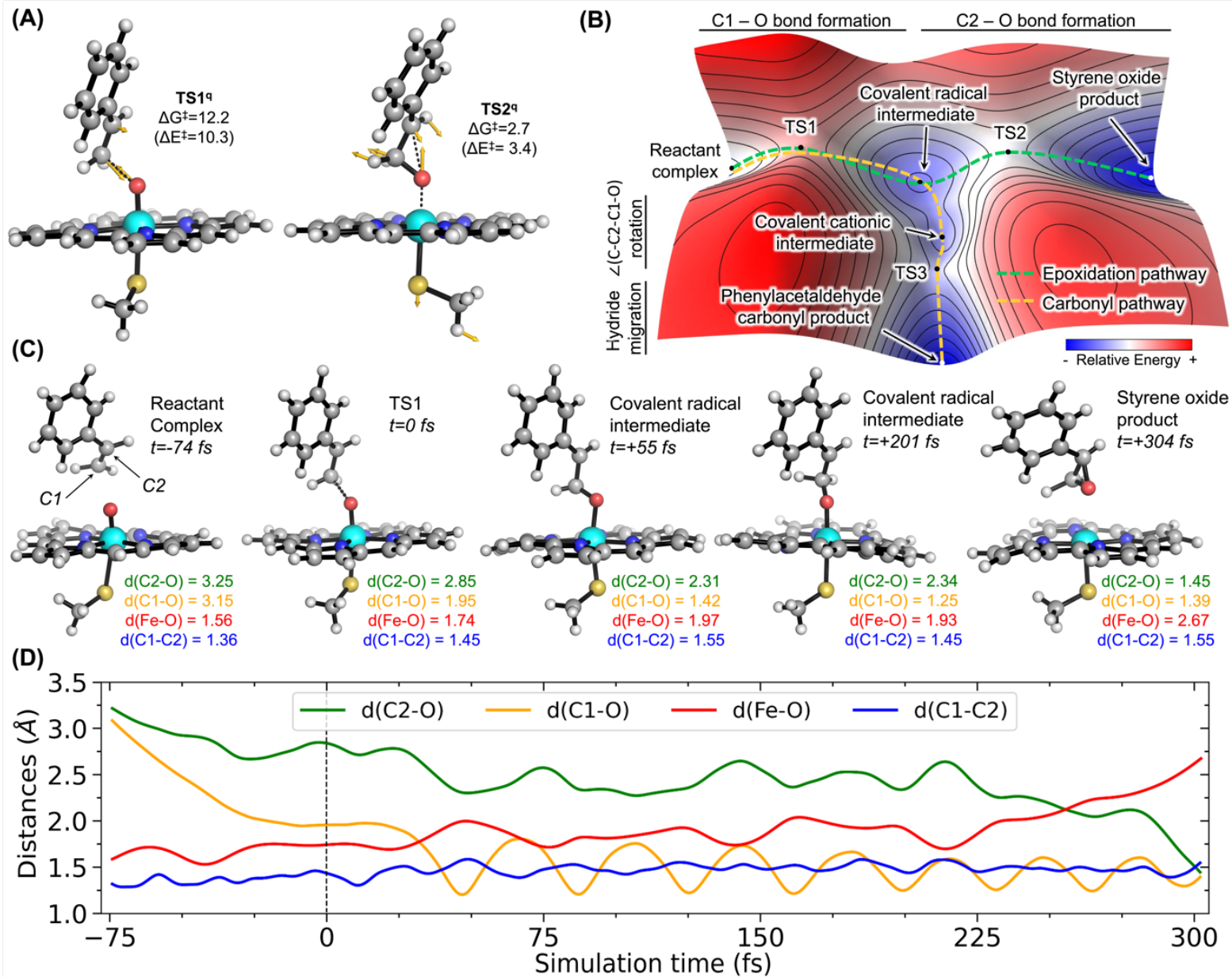

Figure 3. Quasiclassical direct dynamics trajectories (A) Enzyme-free DFT-optimized transition states structures TS1-q (used as starting point for direct QCT simulations) and TS2-q. Yellow arrows are the displacement vectors associated with the transition-state structure imaginary frequency. (B) Qualitative 3D energy landscape with the kinetic selective epoxidation pathway highlighted as green dashed line and the thermodynamic selective carbonyl pathway in orange dashed line. (C) Selected snapshots along a quartet quasiclassical trajectory from reactant complex to styrene oxide formation. (D) Time evolution of most relevant distances along quasiclassical trajectory shown in (C). Energies, distances and time are given in $\mathrm{kcal} \cdot \mathrm{mol}^{-1}$, Angstroms $(\AA)$, and femtoseconds (fs), respectively.

An example of a reactive trajectory on the quartet electronic state for epoxidation is depicted in Fig 3C and 3D (see Fig. S6 for doublet electronic state results). From QTC simulations, the lifetime of the intermediate, defined as the time gap between C1-O and C2O bond formation, is estimated to be ca. $265 \mathrm{fs}$ for the quartet electronic state (ca. $92 \mathrm{fs}$ in the doublet state).

Houk and co-workers proposed a timing criterion to distinguish between dynamically concerted and dynamically stepwise mechanisms. ${ }^{37,43}$ According to this criterion, a mechanism is considered as dynamically concerted if all the bonding changes are completed within less than $60 \mathrm{fs}$ timegap, while it is considered as dynamically stepwise otherwise. This dynamic criterion is complementary to the common definition of concerted vs. stepwise mechanisms based on the shape of the potential energy surface (having one or two potential energy barriers). In this framework, QTC simulations suggest that the epoxidation pathway 
(and the carbonyl formation pathway) in both the quartet and doublet electronic states can be classified as dynamically stepwise mechanisms, although they are potential-energy stepwise and concerted, respectively.

Geometrically concerted and asynchronous, but dynamically stepwise reactions often involve the formation of entropic intermediates. ${ }^{37}$ Entropic intermediates are an ensemble of dynamical structures that reside in a shallow free-energy well, which correspond to a relatively flat region on the potential energy surface (PES) but not to a local minima. These entropic intermediates require to decrease their entropy to exit from this region of the PES. Considering this, QTC simulations are suggesting the existence of an entropic intermediate formed along the doublet PES, after TS1 and prior epoxide product formation, although an intermediate structure could not be optimized as a minimum on the PES (Fig. 2a). This indicates that the carbonyl forming pathway could also be accessible from the doublet electronic state in a nonIRC pathway; when this entropic intermediate is formed, it could give access to the carbocation intermediate if properly stabilized (see the results for the calculated enzymatic reaction in next sections).

While statistical transition state theory, based on static DFT calculations, failed to sufficiently explain the experimentally observed chemoselectivity, reaction dynamic calculations confirmed the strong preference for epoxidation due to dynamic effects. Quasiclassical trajectories revealed that the direction of the atomic motions and momentum acquired right after surpassing TS1 (formation of the first C1-O bond) are strongly coupled to the atomic motions required to overcome the low energy barrier in TS2 (epoxide formation) (Fig. 3C,D). QCT showed that the kinetic energy acquired by the radical intermediate formed from TS1 largely influences its lifetime and dynamically primes the reaction for threemembered ring formation, explaining the origin of the observed high chemoselectivity for epoxidation. Simulations also indicated that this dynamic preference for epoxidation is present whether there is a small potential energy barrier (quartet electronic state, TS2) or not (doublet electronic state).

This "dynamic match" between the TS1 and TS2 reaction coordinates favors epoxidation and is illustrated in Fig. 3B through a qualitative PES. The preference for epoxidation caused by the intrinsic dynamic behavior following TS1 could in fact explain the preferred alkene epoxidation for many catalysts that utilize high-valent iron-oxo (or metal-oxo) species as oxidant. In contrast, the carbonyl pathway requires a specific $\mathrm{C} 1-\mathrm{C} 2$ bond rotation to efficiently stabilize the carbocation intermediate and to allow a 1,2-hydride migration. Due to stereoelectronic effects described above, carbonyl formation depends on very specific conformations of the reactive intermediates that are not dynamically supported. But how did directed enzyme evolution reverse the chemoselectivity in this reaction towards carbonyl formation? 
An important conclusion from DFT calculations and QCTs using a truncated model is that many natural enzymes, including cytochrome P450 monooxygenases ${ }^{13}$ and peroxygenases ${ }^{14}$, achieve selective alkene epoxidation because they exploit the intrinsic preference dictated by the existing "dynamic match" between the two consecutive, dynamically stepwise, C-O bond formations. Consequently, an enzyme (or a catalyst) that aims to produce carbonyls, must overwrite this intrinsic dynamic preference, potentially by allowing the kinetically activated radical intermediate to reach thermal equilibration or by imposing geometric constraints to control the accessible geometries for the intermediate species and transition states.

\section{Computational modelling of the enzyme-substrate bound complex.}

The carbonyl-selective biocatalyst (aMOx) was evolved in ten rounds of directed evolution from a cytochrome P450 enzyme (P450 LA1). ${ }^{23}$ While P450 ${ }_{\text {LA1 }}$ oxidized styrene with low activity (100 TTN) and 55\% epoxide selectivity, aMOx oxidized styrenes with high activity (up to 4500 TTN) and chemoselectivity (up to $94 \%$ carbonyl product). During laboratory evolution, twelve mutations were introduced. Most of these mutations contribute to change chemoselectivity (Fig. 4A) and the mutations scatter over the heme domain of the protein.

To understand how aMOx controls chemoselectivity, we computationally modelled both wildtype P450 ${ }_{\mathrm{LA} 1}$ and the evolved aMOx variant. Since no protein crystal structure is available for P450 $\mathrm{LA} 1$, computational homology models were generated for both enzymes based on a recently solved structure of $\mathrm{P}_{450}$ TT (PDB: $6 \mathrm{GII}$ ) that has $56 \%$ of sequence identity for the heme domain (see SI for details). The models were further refined by performing long-timescale molecular dynamics (MD) simulations in the holo state (5 replicas of $1.0 \mu$ s each, a total of $5.0 \mu \mathrm{s}$ of accumulated simulation time). Analysis and comparison of both active sites revealed that the introduced mutations completely reshaped and narrowed the substrate binding pocket (Fig. 4A). The introduced mutations generated a confined environment that largely reduced the accessible styrene substrate conformations, as confirmed by substrate-bound simulations (Fig. 4B, and Figs S8 and S9). Docking calculations were performed on the most representative structures obtained from holo state MD simulations after clustering analysis based on protein backbone RMSD. Docking predictions were then used as starting points for extensive restrained-MD simulations, in which the distance between the center of mass of the alkene in styrene (defined by $\mathrm{C} 1$ and $\mathrm{C} 2$ atoms) and the oxygen atom from Cpd I was kept restrained during the MD simulation (5 replicas of $500 \mathrm{~ns}$ each, a total of 2,500 ns of restrained-MD simulation time for each system, see computational details). This allowed to explore catalytically relevant binding poses, where the substrate arranges in near attack conformations to carry out the oxidation reaction, largely refining the docking predictions and preventing undesired unbinding events during the simulations. These simulations showed that P450 LA1 can orient styrene towards the iron-oxo species, exposing both enantiotopic faces (re 
and si face), with a preference for si face exposure (Fig. 4B-C and Fig. S10). In contrast, aMOx binds styrene in a reactive mode that preferentially exposes the re face of the alkene (Fig. 4B-C and Fig. S11). Consequently, restrained-MD simulations qualitatively predict an $(R)$-selective epoxide formation from P450 $\mathrm{LA} 1$ wildtype, while the epoxide formation as side product in aMOx variant is predicted to be $(S)$-selective due to the reshaped active site that reaccommodates the styrene substrate and preorganizes it for a selective oxidation.
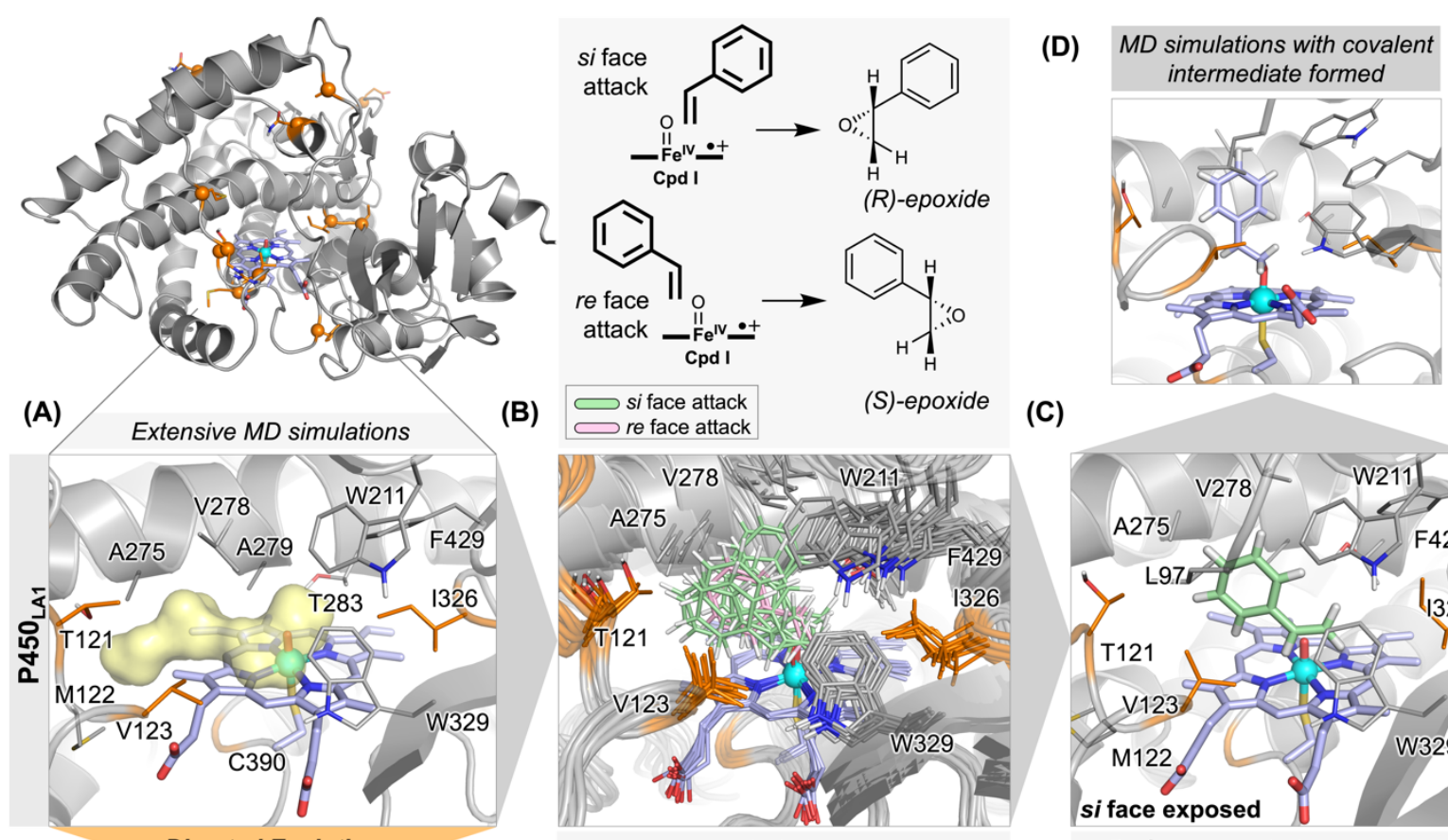

(B) $\rightleftarrows$ si face attack

(S)-epoxide

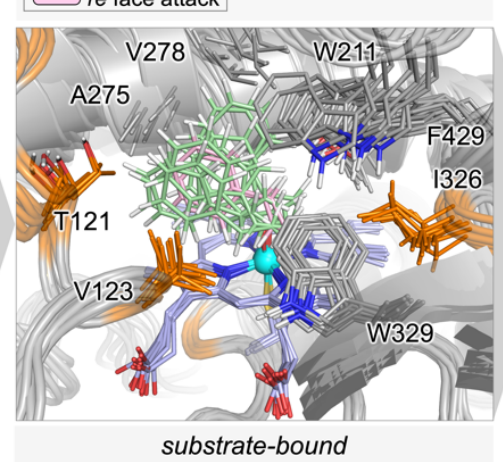

(C)
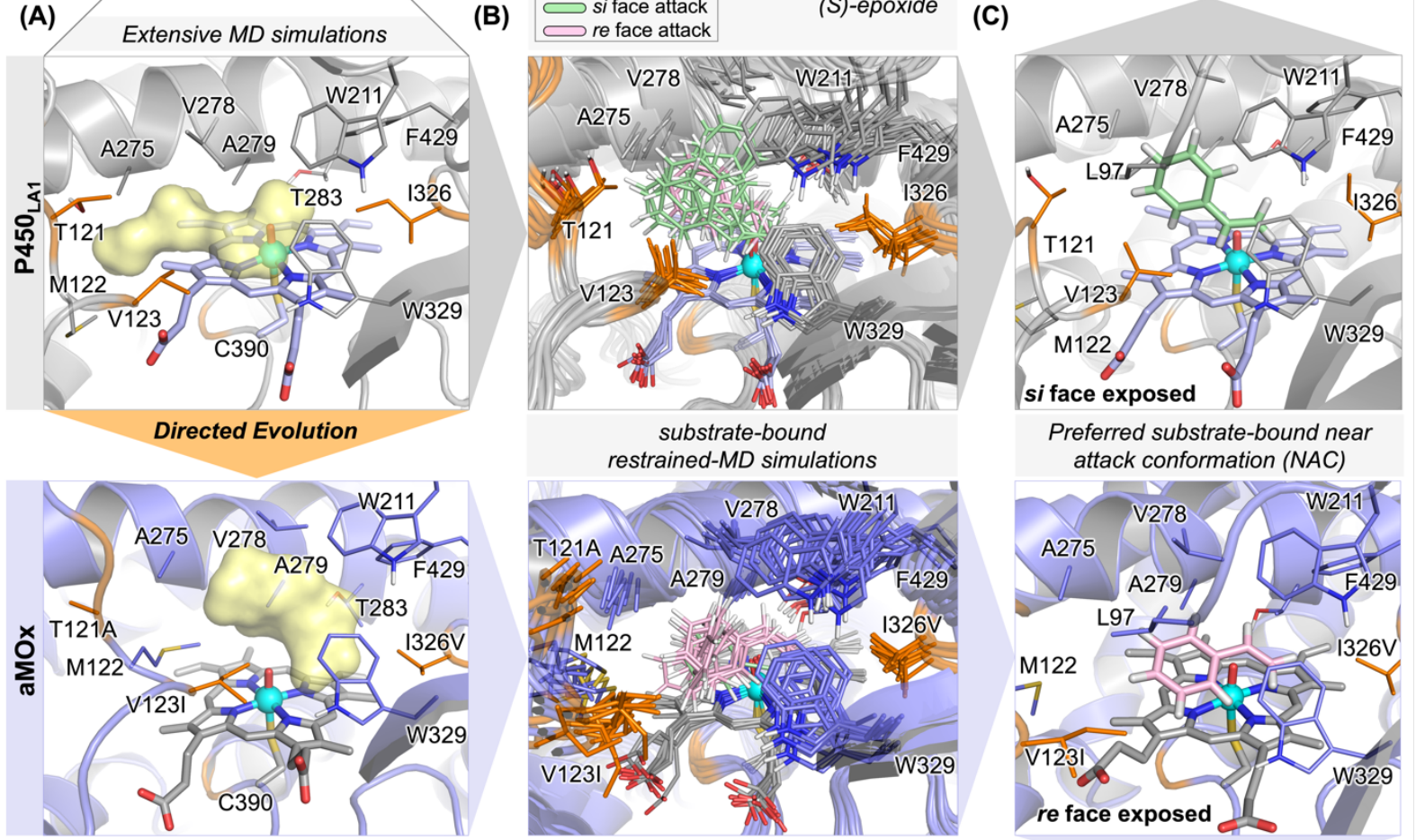

Preferred substrate-bound near attack conformation (NAC)
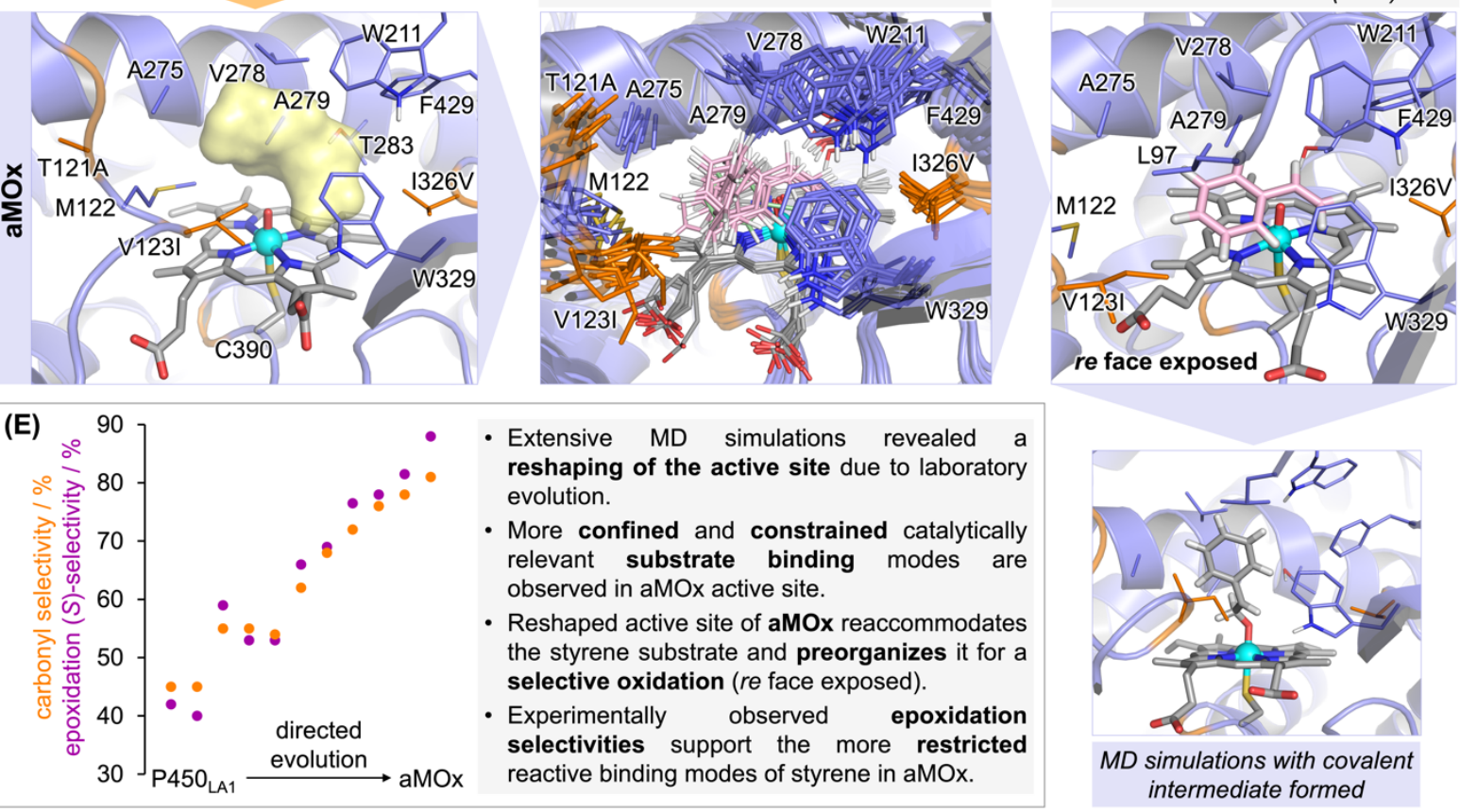

Figure 4. Impact of the directed evolution experiment on the enzyme active site. (A) A homology model was generated for wildtype P450LA1 (56\% sequence identity with template, PDB: 6GII) and aMOx (12 mutations with respect to the wildtype). The homology model was refined by extensive molecular dynamics (MD) simulations 
( $5.0 \mu$ s of accumulated simulation time). Positions mutated along directed evolution are shown as orange balls and stick representation. Active site accessible volume is represented by a yellow surface in the most populated cluster characterized from holo state MD simulations of wildtype P450 $\mathrm{LA}_{1}$ and aMOx enzymes (see also Figs. S8 and S9). (B) Overlay of 10 snapshots of reactive binding modes characterized from restrained-MD simulations of bound styrene in wildtype P450 LA1 and aMOx enzymes (snapshots were arbitrarily selected from intervals of $50 \mathrm{~ns}$ from 500 ns restrained-MD trajectories). Green and pink colored styrene substrate represents si face ( $R$-epoxide) and re face (S-epoxide) binding poses, respectively (see also Figs. S10 and S11). Relevant active site residues are shown in sticks and mutated positions are highlighted in orange. (C) Preferential near attack conformations (NAC) of styrene bound in wildtype P450 $\mathrm{LA}$ and aMOx characterized from restrained-MD simulations. (D) Representative binding mode explored by the covalent intermediate Int1 when formed in wildtype P450LA1 and aMOx active sites observed from MD simulations (see SI for details). (E) Enantioselectivity in the epoxidation as a function of directed evolution. Interestingly, the enantioselectivity of the epoxidation changes during evolution stepwise in analogy to the aMOx selectivity. While wildtype P450 LA1 generates preferably the (R)-enantiomer (60:40 of $R: S)$, epoxidation gets more and more $(S)$-selective in the course of directed evolution and reaches a ratio of 14:86 $(R: S)$ for aMOx.

To find support for the computationally identified reshaping of the active site and its impact on the preferential substrate binding and enzyme selectivity, we experimentally analyzed the enantioselectivity in epoxidation along the course of the directed evolution pathway (Fig. 4E). The P450 LA1 wildtype exhibits a slight preference for the formation of the $(R)$-epoxide enantiomer (60:40 R:S) which qualitatively agrees with the reactive binding modes characterized from computational modelling. Interestingly, the enantiomeric ratio gradually increased towards (S)-epoxide formation during evolution. Although the evolved aMOx enzyme generated the epoxide only in low amounts, this byproduct is obtained with high $(S)$ enantioselectivity (Fig. 4E). Formation of the (S)-epoxide with aMOx is consistent with the preferred re face attack observed computationally. Further, the gradual increase in enantioselectivity during the course of evolution supports the more restricted reactive binding modes of styrene observed in the computational models for the final evolved aMOx variant. Taking together, experimental observations and computational modelling describe that directed evolution increased the confinement in the active site and restricted the accessible, catalytically relevant binding modes of the substrate. This suggests that the accessible conformations of the radical and carbocation intermediates involved in the reaction may also be limited in the more evolved active sites (Fig. 4D).

\section{Computational modelling of the enzymatic reaction mechanisms using QM/MM calculations.}

We hypothesized that restricting the accessible conformations of the intermediates could strongly influence their reactivity and the chemoselectivity in the oxo-transfer reaction. Thus, we used hybrid quantum mechanics/molecular mechanics calculations (QM/MM) ${ }^{44}$ to model the competing oxidation pathways occurring in both enzymes. Representative structures of the covalent intermediate formed in wildtype P450 ${ }_{\mathrm{LA} 1}$ and aMOx enzymes have been used as starting point for $\mathrm{QM} / \mathrm{MM}$ calculations (see SI for details). These structures were obtained from short MD trajectories (100 ns) with the covalent intermediate bound in wildtype P450 LA1 and aMOx actives sites (Fig. 4D). Initial models for intermediate-bound MD simulations were built 
from styrene-bound preferential near attack conformations characterized from restrained-MD simulations (Fig. 4C).

In the P450 LA1-catalyzed system, the preferential NAC of the substrate corresponds to a si face $((R)$-epoxide forming) binding mode of the styrene substrate (Fig. 4C). The alternative re face binding mode of styrene, which is accessible in wildtype P450 $\mathrm{LA}_{1}$, has also been considered in the QM/MM modelling finding similar trends (see SI Fig. S13). ${ }^{45}$ The computed QM/MM reaction profile for the P450 ${ }_{\mathrm{LA} 1}$-catalyzed reaction (Fig. 5) compares well to the DFT modelled enzyme-free truncated model system (Fig. 2), with the formation of the first $\mathrm{C} 1-\mathrm{O}$ bond (LA1-TS1) being the rate-limiting step in both doublet and quartet electronic states (Fig. 5A). Interestingly, and in contrast to the truncated model system, the radical intermediate (LA1-Int1) can be optimized as a minimum on the PES in both electronic states. This indicates that confinement favors the stabilization of the radical species from a potential energy perspective (Fig. 5B). Once formed, the radical intermediate LA1-Int1 can lead to the epoxide product through a low in energy transition state (LA1-TS2, $\left.\Delta \mathrm{G}^{\ddagger}=2.0 \mathrm{kcal} \cdot \mathrm{mol}^{-1}\right)$ in the quartet state and through a barrierless step in the doublet state (Fig. 5C). In comparison, the carbonyl pathway is also accessible from radical LA1-Int1 in both electronic states, where carbocation intermediate LA1-Int2 can be formed slightly downhill after a geometric rearrangement similar to the enzyme-free model, leading to the generation of the carbonyl product (3) though a barrierless transition state (LA1-TS3, see Fig. 5B,C). Due to the steric constraints imposed by the active site cavity, the optimized radical LA1-Int1 and carbocation LA1-Int2 intermediates have the phenyl ring occupying the same position in the enzyme active site. The main geometric difference between these two intermediates is the rotation of the methylene group covalently attached to the $\mathrm{O}$-atom, which ensures effective stabilization of the carbocation intermediate as described above (Fig. 5B).

Based on these results, QM/MM calculations suggest that aldehyde side product formation observed in P450 $\mathrm{LA} 1$ may be attributed to the confinement in the enzyme active site that helps to stabilize the radical intermediate LA1-Int1. However, although the carbonyl pathway is energetically downhill and highly favorable, the epoxide (2) can still be formed as major product, favored by the existing "dynamic match" between the two $\mathrm{C}-\mathrm{O}$ bond forming coordinates. The carbonyl pathway is disfavored by the geometric rearrangement required to form carbocation LA1-Int2 from LA1-Int1 and the fact that epoxide formation from LA1-Int1 is barrierless (doublet) or has a very low energy barrier (quartet LA1-TS2). Consequently, a higher control over the accessible conformations of the nascent radical intermediate would be required to selectively promote the carbonyl pathway. 
(A)

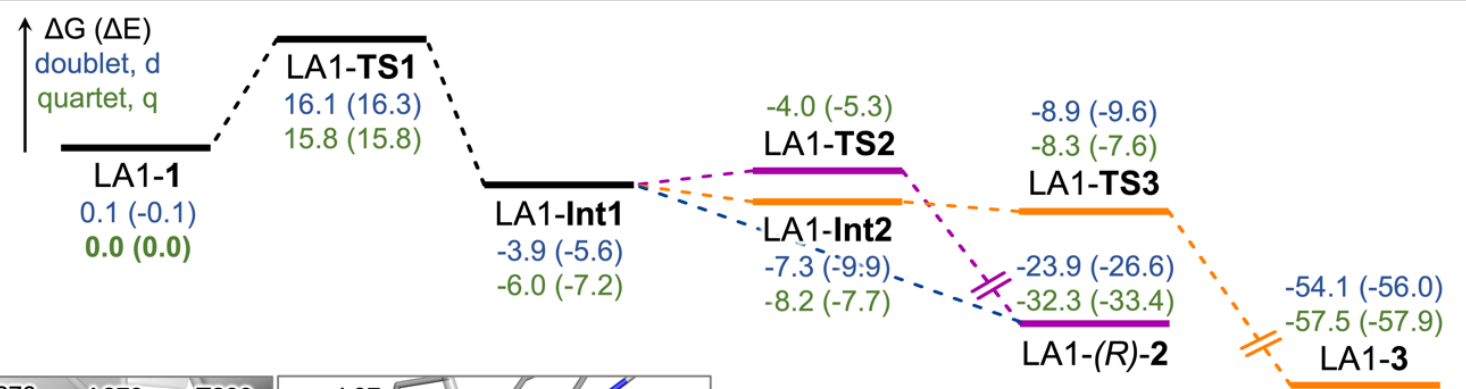

(B)

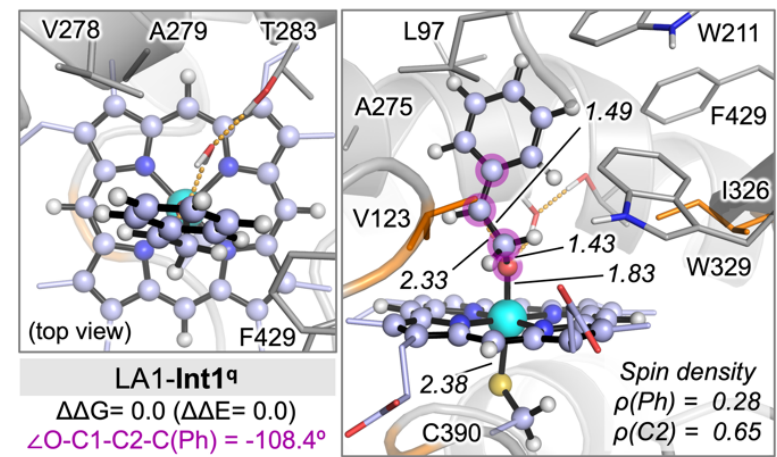

(C)

Epoxide formation in $\mathbf{P 4 5 0}_{\text {LA1 }}$ active site is geometrically favored

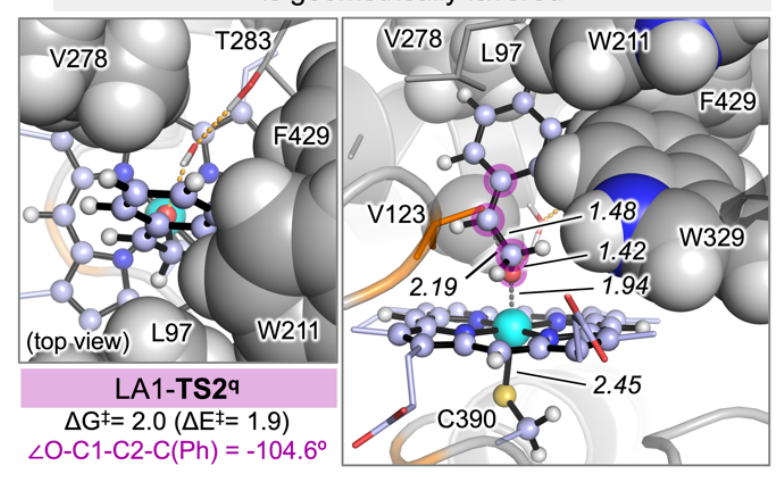

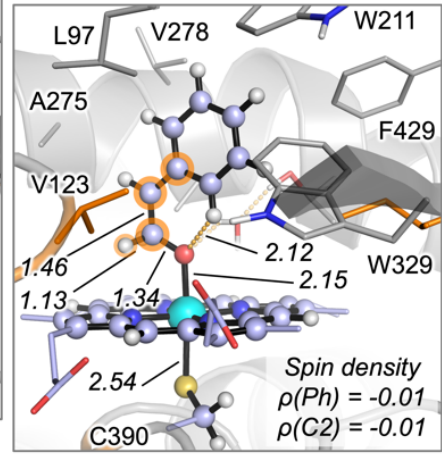

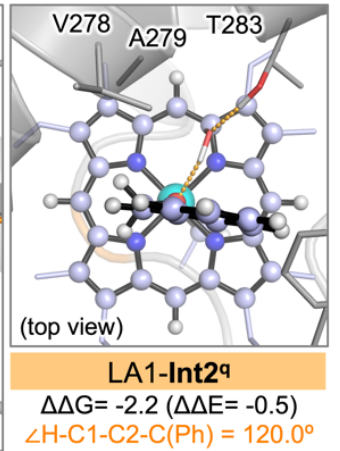

Int2 formation in $\mathbf{P 4 5 0} \mathbf{L A}_{\text {1 }}$ active site can compete with epoxidation, but requires the rotation of Int1 $\mathrm{CH}_{2}$ group

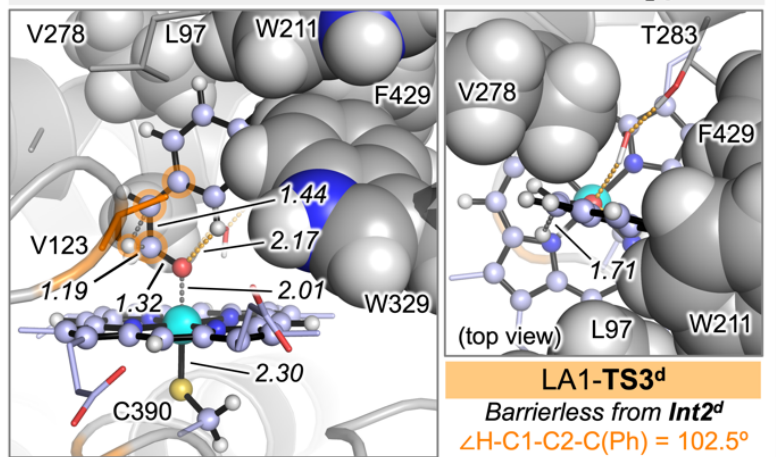

Figure 5. QM/MM calculations on P450 LA1-catalyzed reaction pathways. (A) QM/MM calculated reaction mechanism for the $(S)$-selective epoxidation and carbonyl formation pathways of styrene catalyzed by P450LA1 (see also Fig. S12). A representative snapshot from intermediate-bound MD simulations with styrene in the preferred reactive si face binding pose is used as starting point (see Fig. 4D). Relative Gibbs free energies and electronic energies ( $\Delta \mathrm{G}$ and $\Delta \mathrm{E}$, respectively) are shown in blue for the doublet (d) electronic state and green for the quartet (q). Lowest in energy QM/MM optimized structures for key (B) intermediates and (C) transition states are shown. Space-filling representations for key residues are used to highlight important steric constraints occurring in the active site. Energies, distances and angles are given in $\mathrm{kcal} \cdot \mathrm{mol}^{-1}$, angstroms $(\AA)$ and degrees $\left(^{\circ}\right)$, respectively. QM/MM calculated mechanism based on a re face near attack conformation of styrene in P450 LA1 active site is reported in Fig. $\mathbf{S 1 3 .}$

In a next step, we calculated the competing carbonyl forming and epoxidation reaction pathways catalyzed by the evolved aMOx biocatalyst (Fig. 6). These QM/MM calculations revealed significant differences compared to the truncated model (Fig. 2) and P450LA1 (Fig. 5). QM/MM calculations were carried out considering the preferred reactive binding mode of styrene in the aMOx active site (Fig. 4), where the re face of the alkene is exposed to the ironoxo active species (see SI Fig. S13 for calculations involving the alternative minor si-face binding mode ${ }^{45}$ which exhibit significantly higher reaction barriers and thus making it 
significantly less reactive). These calculations showed that, similarly to the wildtype P450 system, both doublet and quartet radical intermediates aMOx-Int1 can be optimized and characterized as minima on the potential energy surface (Fig. 6A). Additionally, calculations described a significant barrier for the second C2-O bond formation (aMOx-TS2, Fig. 6A) from the radical intermediate aMOx-Int1 in both doublet or quartet electronic states. The estimated barriers for three-membered ring formation (aMOx-TS2, $\Delta \mathrm{G}^{\ddagger}=5.7$ and $10.7 \mathrm{kcal} \cdot \mathrm{mol}^{-1}$ on the doublet and the quartet PES, respectively) are significantly higher as compared to the ones estimated for the enzyme-free model (Fig. 2) and wildtype enzyme (Fig. 5). This observation presents a scenario in which the radical intermediate is stabilized by the active site of aMOx due to confinement and steric interactions increasing its lifetime (Fig. 6A-B). This leads to an effective thermal equilibration, loss of the excess of kinetic energy and vibrational energy redistribution. The reactive radical intermediate can thus follow the lowest in energy reaction pathway to generate the carbonyl product. QM/MM calculations describe that the carbocation intermediate formation is uphill (aMOx-Int2, $\Delta \Delta G=2.2$ and $9.1 \mathrm{kcal} \cdot \mathrm{mol}^{-1}$ on the doublet and the quartet PES, respectively), but these carbocation intermediates are lower in energy than the corresponding epoxide forming transition states (Fig. 6A). 1,2-Hydride migration from carbocation aMOx-Int2 are found to be barrierless in both electronic states (Fig. 6A and C), with the doublet electronic state pathway being lower in energy than the quartet electronic state pathway. Tight active site packing in the aMOx variant stabilizes the radical intermediate by holding the aromatic ring in a specific position establishing hydrophobic interactions with active site residues L97, V123I, V278 and A279 (Fig. 6B-C). Similar to wildtype P450 LA1, formation of the carbocation involves the rotation of the methylene-oxygen group to ensure stereoelectronic stabilization of the carbocation while the aromatic ring is held at the same position (Fig. 6B).

Thus, aMOx can override the intrinsic dynamic preferences for epoxidation by imposing steric constraints to the formed radical intermediate. This disfavors the epoxidation TS2 with respect to the carbocation formation and hydride migration transition state TS3. Carbonyl formation is thus energetically more favorable than epoxidation which explains the chemoselectivity achieved by the evolved aMOx enzyme. 
(A)

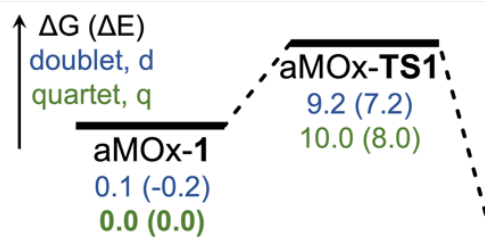

(B)
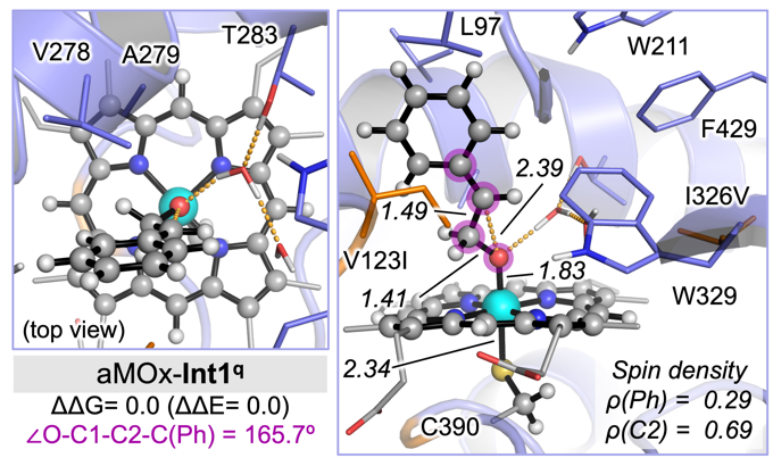

(C)

Steric constraints on Int1 by aMOx active site disfavor epoxide forming TS2
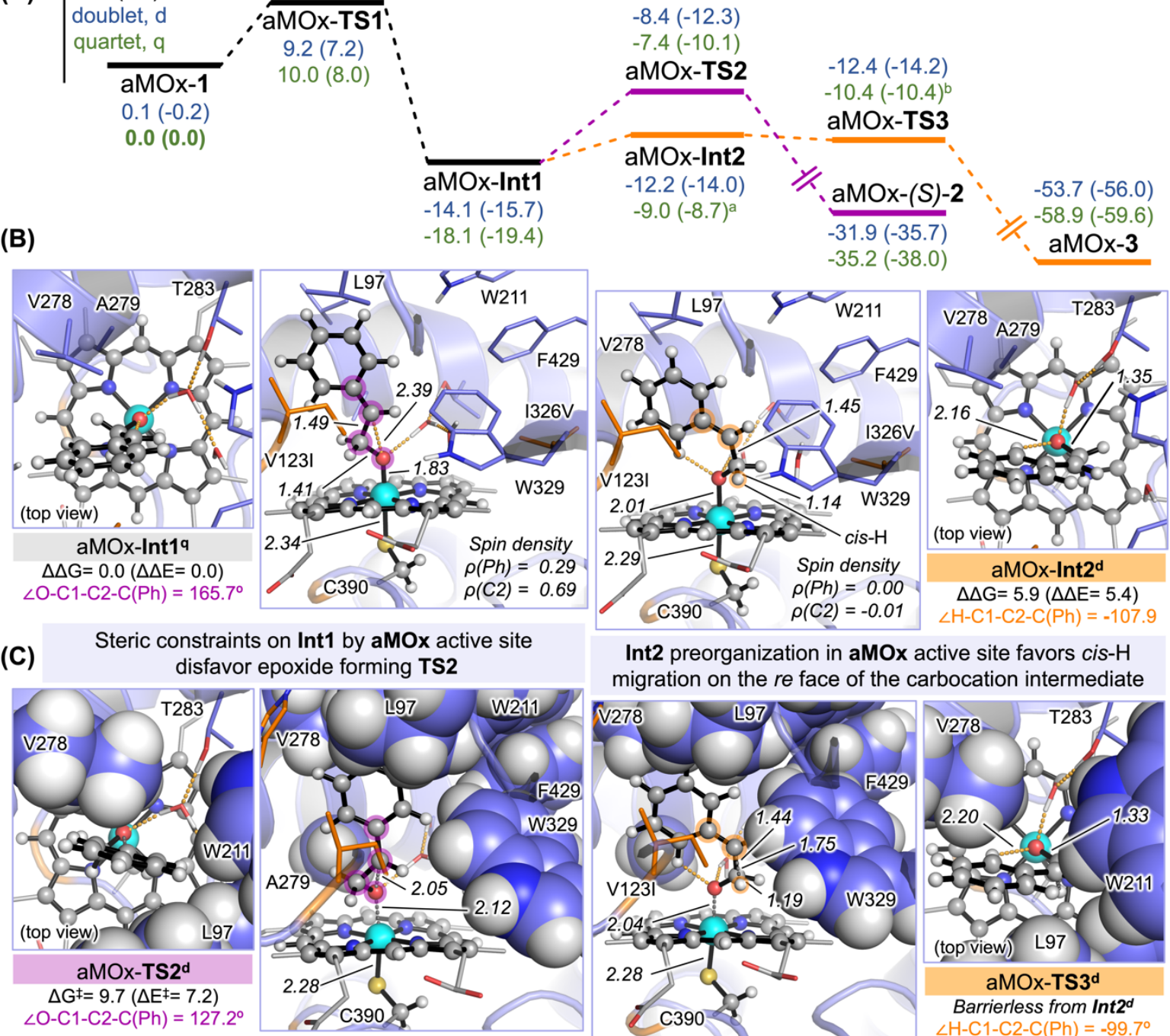

Int2 preorganization in aMOx active site favors cis- $\mathrm{H}$ migration on the re face of the carbocation intermediate
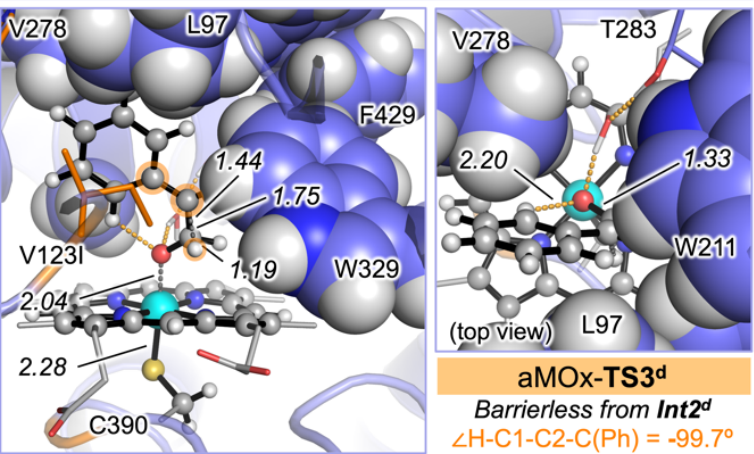

Figure 6. QM/MM calculations on aMOx-catalyzed reaction pathways. (A) QM/MM calculated reaction mechanism for the (S)-selective epoxidation and carbonyl formation pathways of styrene catalyzed by the aMOx variant (see also Fig. S14). A representative snapshot from intermediate-bound MD simulations with styrene in the preferred reactive re face binding pose is used as starting point (see Fig. 4D). Relative Gibbs free energies and electronic energies $(\Delta \mathrm{G}$ and $\Delta \mathrm{E}$, respectively) are shown in blue for doublet (d) electronic state and green for quartet (q). Lowest in energy QM/MM optimized structures for key $(\mathbf{B})$ intermediates and $(\mathbf{C})$ transition states are shown. Space-filling representations for key residues are used to highlight important steric constraints occurring in the active site. Energies, distances and angles are given in $\mathrm{kcal} \cdot \mathrm{mol}^{-1}$, angstroms $(\AA)$ and degrees $\left({ }^{\circ}\right)$, respectively. $\mathrm{QM} / \mathrm{MM}$ calculated mechanism based on the minor explored si face near attack conformation of styrene in aMOx active site is reported in Fig. S15. a Structure optimized with $\mathrm{C} 2-\mathrm{cis}-\mathrm{H}$ and $\mathrm{C} 2-$ trans-H distances frozen. Distance values are taken from optimized structure in the doublet state. Frequency calculation showed that the optimized structure has all frequencies positive. ${ }^{\mathrm{b}}$ TS structure optimized with $\mathrm{C} 2-\mathrm{cis}-\mathrm{H}, \mathrm{C} 1-\mathrm{cis}-\mathrm{H}$ and $\mathrm{O}-\mathrm{C} 1$ distances frozen. Distance values are taken from optimized structure in the doublet state. Frequency calculation showed that all frequencies of the optimized structure are positive except one, which corresponds to the $\mathrm{H}$-migration coordinate.

\section{Selective hydride migration: Synergies between computational modelling and enzymatic conversion of deuterium labeled styrenes.}

QM/MM calculations do not only reveal that the protein environment offers a high degree of conformational control over the reactive intermediates, but also propose a controlled stereoselective hydride migration. The tight conformational control of aMOx primes the cis- $\mathrm{H}$ 
atom of the styrene substrate for selective migration on the re face of the carbocation intermediate through the lowest in energy transition state aMOx-TS3 (Fig. 6B-C and Fig. S14). This can be rationalized by the oxygen attack from the re face and rotation of the C1-C2 bond to generate a stabilized carbocation intermediate, which geometrically preorganizes the cis- $\mathrm{H}$ for migration by aligning this $\mathrm{C}-\mathrm{H}$ bond with the empty $p$-orbital of the benzylic carbocation (Fig. 6B).

To validate the predictions obtained from $\mathrm{QM} / \mathrm{MM}$ calculations and to further support the modus operandi of the carbonyl-selective aMOx enzyme, we synthesized deuterium-labelled cis- as well as trans-styrene-( $\beta)$ - $d$ according to literature. ${ }^{46,47}$ Both substrates were independently converted by the aMOx enzyme. To avoid loss of the deuterium label by ketoenol tautomerism of the aldehyde product, the reactions were performed in an enzyme cascade together with a phenylacetaldehyde reductase (PAR). We used mass spectrometry to characterize the position of the deuterium-label in the products (Fig. S16). While hydride migration could be observed with deuterium label at trans-position, deuteride migration occurred with the cis-labeled styrene (Fig. 7). The experimental data are in full agreement with the computational investigations and demonstrate that aMOx catalyzes a cis-selective 1,2-migration.

(A)

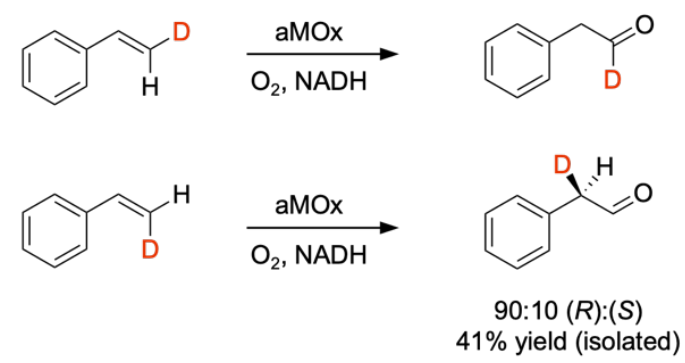

(B)

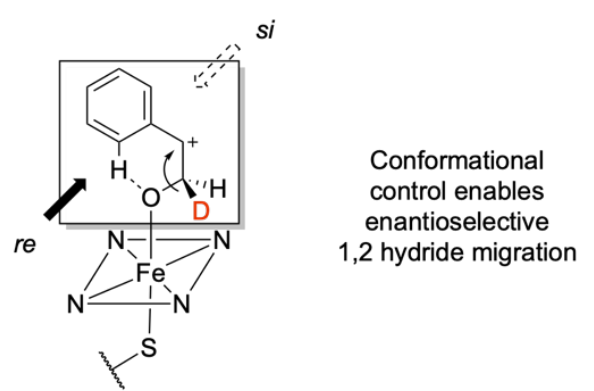

Figure 7. (A) Determination of the cis/trans- as well as stereoselectivity of the 1,2-hydride migration with deuteriumlabeled styrenes as substrates. (B) Conformational control of the carbocation intermediate allows an enantioselective 1,2-migration yielding the aldehyde as $(R)$-enantiomer predominantly.

Finally, we aimed to analyze the enantioselectivity during 1,2-hydride migration. Computational as well as recent experimental studies ${ }^{23}$ suggested that aMOx can control the enantioselectivity in the 1,2-hydride migration. According to our QM/MM calculations the cishydride migrates selectively on the re face of the prochiral substrate due to conformational restraints imposed by the active site of the enzyme and the reactive binding pose that the substrate and intermediates adopt (see above). To support this finding with experimental evidences, we performed a preparative scale reaction with cis-styrene-( $\beta)$ - $d$ as substrate and aMOx as catalyst (Fig. S17, S18). The upscaling was performed in a cascade reaction with PAR to avoid racemization of the aldehyde via keto-enol tautomerism. The deuterium-labeled 
alcohol was isolated with a yield of $41 \%$ and converted to the Mosher ester. ${ }^{1} \mathrm{H}$ NMR analysis of the ester revealed that the formed stereocenter is predominantly $(R)$-configurated with a selectivity of $>90: 10(R):(S)$. Consequently, the 1,2-hydride/deuteride migration occurs selectively on the re-side which is in full agreement with computational models and predictions. It is currently not clear whether the $<10 \%(S)$-enantiomer derives from racemization of the aldehyde or by unselective 1,2-migration.

Overall, this strengthens our findings that aMOx is the first catalyst capable of performing enantioselective alkene to carbonyl oxidation. aMOx achieves this by a catalytic enantioselective 1,2-hydride migration, which has to the best of our knowledge not been reported so far. Catalytic enantioselective 1,2-migrations of substituents on prochiral carbocations are very challenging and up to now limited to migration of alkyl or phenyl groups, often in ring-strain releasing processes. ${ }^{48-50}$ Stereoselective 1,2-migrations of hydrides are known, but typically restricted to chiral, cyclic molecules. In this substrate-controlled processes the stereochemical information is already encoded in the structure of the starting material. ${ }^{51}$ In contrast, aMOx achieves enantioselectivity by catalyst-control, a feature that can potentially be used to control many other molecular rearrangement reactions ${ }^{52}$ involving reactive intermediates. Overall, the cis- as well as enantioselective 1,2-hydride migration is not only a remarkable catalyst-controlled process, but also supports the computational predictions on the enzymatic mechanism.

\section{Conclusions}

A combination of multiscale computational methods have been employed to model the oxidation of styrene by heme Fe-oxo active species in the absence of any enzyme scaffold and in an enzymatic environment specifically evolved to carry out a carbonyl-selective oxidation reaction. DFT calculations for styrene as substrate indicated that the epoxidation pathway and the direct oxidation to an aldehyde (carbonyl forming pathway) are energetically accessible, exhibiting low intrinsic barriers, and diverge after the first $\mathrm{C} 1-\mathrm{O}$ bond formation that leads to a covalent radical intermediate. DFT calculations and direct quasiclassical trajectories showed that the fleeting radical intermediate is formed with a large excess of kinetic energy. In addition, these calculations revealed a strong coupling between the reaction coordinate that describes this first $\mathrm{C} 1-\mathrm{O}$ bond formation with the coordinate that promotes the second $\mathrm{C} 2-\mathrm{O}$ bond formation. These factors intrinsically favor the epoxide product formation over the aldehyde formation in the enzyme-free model system. However, when the reaction takes place in an enzymatic framework, confinement helps on dissipating the excess of energy of the radical intermediate, allowing its thermal equilibration. In addition, QM/MM calculations 
described that the evolved aMOx enzyme imposes steric constraints to the reactive intermediates when formed in its active site which limits their accessible conformations. This translates into disfavoring the transition state that leads to the epoxide product via second C2-O bond formation, while allowing a cis- and enantioselective 1,2-hydride migration to preferentially generate the aldehyde product. Consequently, intrinsic dynamic preferences and the important "dynamic match" that strongly favors epoxidation are overridden by the evolved aMOx enzyme. These computational predictions were further supported by experimental investigations using deuterated styrene substrates, demonstrating that confinement achieved by the evolved aMOx enzyme also controls the stereoselectivity in the hydride-migration step.

Our results demonstrate that confinement ${ }^{34}$ is essential to alter and control the intrinsic dynamic behavior of these highly reactive fleeting intermediates formed over the course of these diverging oxidation pathways. The behavior of such intermediates, which indeed can be (pro-)chiral, ${ }^{45}$ finally determines the chemoselectivity of the reaction. Mechanistic insights obtained in this work provide useful guidance that may help to further expand this new biocatalytic direct oxidation of alkenes to carbonyl compounds towards more challenging substrates, including internal alkenes ${ }^{53}$ or unactivated, aliphatic alkenes. We envision that other biological or abiological enzyme-catalyzed reactions might use similar mechanisms to outcompete intrinsic dynamic effects of reactive intermediates to access alternative catalytic cycles that are challenging to achieve otherwise. We expect that our study paves the way towards the design of new enzyme-catalyzed reactions that exploit these features.

\section{Acknowledgements}

This work was supported by the Spanish MICINN (Ministerio de Ciencia e Innovación) PID2019-111300GA-I00 project and Juan de la Cierva-Incorporación fellowship IJCI-201733411 (M.G.B), the Generalitat de Catalunya AGAUR Beatriu de Pinós H2020 MSCACOFUND (grant agreement No 801370) 2018-BP-00204 project (M.G.B.), the Spanish MIU (Ministerio de Universidades) predoctoral FPU fellowship FPU18/02380 (J.S.) and the Deutsche Forschungsgemeinschaft via the Emmy Noether fellowship 420112577 (S.C.H.). The authors thankfully acknowledge the computer resources and the technical support provided by Barcelona Supercomputing Center BSC-RES (RES-QSB-2019-3-0009, RESQSB-2020-2-0016, and RES-QH-2021-2-0013). 


\section{References}

(1) Dong, J. J.; Browne, W. R.; Feringa, B. L. Palladium-Catalyzed Anti-Markovnikov Oxidation of Terminal Alkenes. Angew. Chem. Int. Ed. 2015, 54, 734-744. https://doi.org/10.1002/anie.201404856.

(2) Morandi, B.; Wickens, Z. K.; Grubbs, R. H. Practical and General Palladium-Catalyzed Synthesis of Ketones from Internal Olefins. Angew. Chem. Int. Ed. 2013, 52 (10), 2944-2948. https://doi.org/10.1002/anie.201209541.

(3) Bode, J. W.; Mahatthananchai, J.; Dumas, A. M. Catalytic Selective Synthesis. Angew. Chem. Int. Ed. 2012, 51, 10954-10990. https://doi.org/10.1002/anie.201201787.

(4) Jørgensen, K. A. Transition-Metal-Catalyzed Epoxidations. Chem. Rev. 1989, 89 (3), 431-458.

(5) Holm, R. H. Metal-Centered Oxygen Atom Transfer Reactions. Chem. Rev. 1987, 87 (6), 14011449. https://doi.org/10.1021/cr00082a005.

(6) Zhang, W.; Loebach, J. L.; Wilson, S. R.; Jacobsen, E. N. Enantioselective Epoxidation of Olefins Catalyzed by (Salen)Manganese Complexes. J. Am. Chem. Soc. 1990, 112 (7), 28012803. https://doi.org/10.1021/ja00163a052.

(7) Meunier, B.; de Visser, S. P.; Shaik, S. Mechanism of Oxidation Reactions Catalyzed by Cytochrome P450 Enzymes. Chem. Rev. 2004, 104 (9), 3947-3980. https://doi.org/10.1021/cr020443g.

(8) Jacobsen, E. N.; Zhang, W.; Muci, A. R.; Ecker, J. R.; Deng, L. Highly Enantioselective Epoxidation Catalysts Derived from 1,2-Diaminocyclohexane. J. Am. Chem. Soc. 1991, 113 (18), 7063-7064. https://doi.org/10.1021/ja00018a068.

(9) Che, C.-M.; Huang, J.-S. Metalloporphyrin-Based Oxidation Systems: From Biomimetic Reactions to Application in Organic Synthesis. Chem. Commun. 2009, No. 27, 3996-4015. https://doi.org/10.1039/b901221d.

(10) Costas, M.; Mehn, M. P.; Jensen, M. P.; Que, L. Dioxygen Activation at Mononuclear Nonheme Iron Active Sites: Enzymes, Models, and Intermediates. Chem. Rev. 2004, 104, 969-986. https://doi.org/10.1021/cr020628n.

(11) Chen, K.; Costas, M.; Kim, J.; Tipton, A. K.; Que, L. Olefin Cis-Dihydroxylation versus Epoxidation by Non-Heme Iron Catalysts: Two Faces of an Felll-OOH Coin. J. Am. Chem. Soc. 2002, 124 (12), 3026-3035. https://doi.org/10.1021/ja0120025.

(12) Cussó, O.; Garcia-Bosch, I.; Ribas, X.; Lloret-Fillol, J.; Costas, M. Asymmetric Epoxidation with $\mathrm{H} 2 \mathrm{O} 2$ by Manipulating the Electronic Properties of Non-Heme Iron Catalysts. J. Am. Chem. Soc. 2013, 135 (39), 14871-14878. https://doi.org/10.1021/ja4078446.

(13) Ortiz De Montellano, P. R. Cytochrome P450: Structure, Mechanism, and Biochemistry; Springer US, 2005. https://doi.org/10.1007/b139087.

(14) Hofrichter, M.; Ullrich, R. Oxidations Catalyzed by Fungal Peroxygenases. Curr. Opin. Chem. Biol. 2014, 19 (1), 116-125. https://doi.org/10.1016/j.cbpa.2014.01.015.

(15) Krebs, C.; Fujimori, D. G.; Walsh, C. T.; Bollinger, J. M. Non-Heme Fe(IV)-Oxo Intermediates. In Accounts of Chemical Research; 2007; Vol. 40, pp 484-492. https://doi.org/10.1021/ar700066p.

(16) Ostovic, D.; Bruice, T. C. Mechanism of Alkene Epoxidation by Iron, Chromium, and Manganese Higher Valent Oxo-Metalloporphyrins. Acc. Chem. Res. 1992, 25 (7), 314-320. https://doi.org/10.1021/ar00019a007.

(17) Linker, T. The Jacobsen - Katsuki Epoxidation and Its Controversial Mechanism. Angew. Chem. Int. Ed. 1997, 36 (19), 2060-2062. https://doi.org/10.1002/anie.199720601.

(18) Groves, J. T.; Myers, R. S. Catalytic Asymmetric Epoxidations with Chiral Iron Porphyrins. J. Am. Chem. Soc. 1983, 105 (18), 5791-5796. https://doi.org/10.1021/ja00356a016.

(19) de Visser, S. P.; Ogliaro, F.; Harris, N.; Shaik, S. Multi-State Epoxidation of Ethene by Cytochrome P450: A Quantum Chemical Study. J. Am. Chem. Soc. 2001, 123 (13), 3037-3047. https://doi.org/10.1021/ja003544. 
(20) de Visser, S. P.; Kumar, D.; Shaik, S. How Do Aldehyde Side Products Occur during Alkene Epoxidation by Cytochrome P450? J. Inorg. Biochem. 2004, 98 (7), 1183-1193. https://doi.org/10.1016/j.jinorgbio.2004.01.015.

(21) Duan, L.; Jogl, G.; Cane, D. E. The Cytochrome P450-Catalyzed Oxidative Rearrangement in the Final Step of Pentalenolactone Biosynthesis: Substrate Structure Determines Mechanism. J. Am. Chem. Soc. 2016, 138, 12678-12689. https://doi.org/10.1021/jacs.6b08610.

(22) Wang, X.; Shi, J.; Liu, Y. Oxidative Rearrangement Mechanism of Pentalenolactone F Catalyzed by Cytochrome P450 CYP161C2 (PntM). Inorg. Chem. 2018, 57 (15), 8933-8941. https://doi.org/10.1021/acs.inorgchem.8b00860.

(23) Hammer, S. C.; Kubik, G.; Watkins, E.; Huang, S.; Minges, H.; Arnold, F. H. Anti-Markovnikov Alkene Oxidation by Metal-Oxo-Mediated Enzyme Catalysis. Science 2017, 358 (6360), 215218. https://doi.org/10.1126/science.aao1482.

(24) Kurouchi, H.; Singleton, D. A. Labelling and Determination of the Energy in Reactive Intermediates in Solution Enabled by Energy-Dependent Reaction Selectivity. Nat. Chem. 2018, 10 (2), 237-241. https://doi.org/10.1038/NCHEM.2907.

(25) Assmann, J.; Charvat, A.; Schwarzer, D.; Kappel, C.; Luther, K.; Abel, B. Real-Time Observation of Intra- and Intermolecular Vibrational Energy Flow of Selectively Excited Alkyl lodides in Solution: The Effect of Chemical Substitution. J. Phys. Chem. A 2002, 106 (21), 5197-5201. https://doi.org/10.1021/jp015552y.

(26) Ess, D. H.; Wheeler, S. E.; lafe, R. G.; Xu, L.; Çelebi-Ölçüm, N.; Houk, K. N. Bifurcations on Potential Energy Surfaces of Organic Reactions. Angew. Chem. Int. Ed. 2008, 47 (40), 75927601. https://doi.org/10.1002/anie.200800918.

(27) Quijano, L. M. M.; Singleton, D. A. Competition between Reaction and Intramolecular Energy Redistribution in Solution: Observation and Nature of Nonstatistical Dynamics in the Ozonolysis of Vinyl Ethers. J. Am. Chem. Soc. 2011, 133 (35), 13824-13827. https://doi.org/10.1021/ja2043497.

(28) Kurouchi, H.; Andujar-De Sanctis, I. L.; Singleton, D. A. Controlling Selectivity by Controlling Energy Partitioning in a Thermal Reaction in Solution. J. Am. Chem. Soc. 2016, 138 (44), 14534-14537. https://doi.org/10.1021/jacs.6b09052.

(29) Yang, B.; Schouten, A.; Ess, D. H. Direct Dynamics Trajectories Reveal Nonstatistical Coordination Intermediates and Demonstrate That $\sigma$ and ?-Coordination Are Not Required for Rhenium(I)-Mediated Ethylene C-H Activation. J. Am. Chem. Soc. 2021, 143 (22), 8367-8374. https://doi.org/10.1021/jacs.1c01709.

(30) Ess, D. H. Quasiclassical Direct Dynamics Trajectory Simulations of Organometallic Reactions. Acc. Chem. Res. 2021, 54 (23), 4410-4422. https://doi.org/10.1021/acs.accounts.1c00575.

(31) Hare, S. R.; Tantillo, D. J. Post-Transition State Bifurcations Gain Momentum-Current State of the Field. Pure Appl. Chem. 2017, 89 (6), 679-698. https://doi.org/10.1515/pac-2017-0104.

(32) Hong, Y. J.; Tantillo, D. J. Biosynthetic Consequences of Multiple Sequential Post-TransitionState Bifurcations. Nat. Chem. 2014, 6 (2), 104-111. https://doi.org/10.1038/nchem.1843.

(33) Hong, Y. J.; Tantillo, D. J. A Potential Energy Surface Bifurcation in Terpene Biosynthesis. Nat. Chem. 2009, 1 (5), 384-389. https://doi.org/10.1038/nchem.287.

(34) Mitschke, B.; Turberg, M.; List, B. Confinement as a Unifying Element in Selective Catalysis. Chem 2020, 6 (10), 2515-2532. https://doi.org/10.1016/j.chempr.2020.09.007.

(35) Shaik, S.; Kumar, D.; de Visser, S. P.; Altun, A.; Thiel, W. Theoretical Perspective on the Structure and Mechanism of Cytochrome P450 Enzymes; 2005; Vol. 105. https://doi.org/10.1021/cr030722j.

(36) Harvey, J. N.; Aschi, M.; Schwarz, H.; Koch, W. The Singlet and Triplet States of Phenyl Cation. A Hybrid Approach for Locating Minimum Energy Crossing Points between Non-Interacting Potential Energy Surfaces. Theor. Chem. Acc. 1998, 99 (2), 95-99. https://doi.org/10.1007/s002140050309.

(37) Yang, Z.; Jamieson, C. S.; Xue, X. S.; Garcia-Borràs, M.; Benton, T.; Dong, X.; Liu, F.; Houk, K. 
N. Mechanisms and Dynamics of Reactions Involving Entropic Intermediates. Trends Chem. 2019, 1 (1), 22-34. https://doi.org/10.1016/j.trechm.2019.01.009.

(38) Hong, Y. J.; Tantillo, D. J. A Potential Energy Surface Bifurcation in Terpene Biosynthesis. Nat. Chem. 2009, 1 (5), 384-389. https://doi.org/10.1038/nchem.287.

(39) Yang, Z.; Yang, S.; Yu, P.; Li, Y.; Doubleday, C.; Park, J.; Patel, A.; Jeon, B. sun; Russell, W. K.; Liu, H. wen; Russell, D. H.; Houk, K. N. Influence of Water and Enzyme SpnF on the Dynamics and Energetics of the Ambimodal [6+4]/[4+2] Cycloaddition. Proc. Natl. Acad. Sci. U. S. A. 2018, 115 (5), E848-E855. https://doi.org/10.1073/pnas.1719368115.

(40) Biswas, B.; Singleton, D. A. Controlling Selectivity by Controlling the Path of Trajectories. J. Am. Chem. Soc. 2015, 137 (45), 14244-14247. https://doi.org/10.1021/jacs.5b08635.

(41) Nieves-Quinones, Y.; Singleton, D. A. Dynamics and the Regiochemistry of Nitration of Toluene. J. Am. Chem. Soc. 2016, 138 (46), 15167-15176. https://doi.org/10.1021/jacs.6b07328.

(42) Roytman, V. A.; Jin, S.; Nguyen, V. T.; Nguyen, V. D.; Haug, G. C.; Larionov, O. V.; Singleton, D. A. Bond Memory in Dynamically Determined Stereoselectivity. J. Am. Chem. Soc. 2020, 142 (1), 85-88. https://doi.org/10.1021/jacs.9b12227.

(43) Yang, Z.; Houk, K. N. The Dynamics of Chemical Reactions: Atomistic Visualizations of Organic Reactions, and Homage to van 't Hoff. Chem. Eur. J. 2018, 24 (16), 3916-3924. https://doi.org/10.1002/chem.201706032.

(44) Romero-Rivera, A.; Garcia-Borràs, M.; Osuna, S. Computational Tools for the Evaluation of Laboratory-Engineered Biocatalysts. Chem. Commun. 2016, 53, 284-297. https://doi.org/10.1039/C6CC06055B.

(45) Reetz, M. T.; Garcia-Borràs, M. The Unexplored Importance of Fleeting Chiral Intermediates in Enzyme-Catalyzed Reactions. J. Am. Chem. Soc. 2021, 143 (37), 14939-14950. https://doi.org/10.1021/jacs.1c04551.

(46) Ball, L. T.; Lloyd-Jones, G. C.; Russell, C. A. Gold-Catalysed Oxyarylation of Styrenes and Mono- and Gem -Disubstituted Olefins Facilitated by an lodine(III) Oxidant. Chem. Eur. J. 2012, 18 (10), 2931-2937. https://doi.org/10.1002/chem.201103061.

(47) Wei, Y.; Tinoco, A.; Steck, V.; Fasan, R.; Zhang, Y. Cyclopropanations via Heme Carbenes: Basic Mechanism and Effects of Carbene Substituent, Protein Axial Ligand, and Porphyrin Substitution. J. Am. Chem. Soc. 2018, 140 (5), 1649-1662. https://doi.org/10.1021/jacs.7b09171.

(48) Liang, T.; Zhang, Z.; Antilla, J. C. Chiral Brønsted Acid Catalyzed Pinacol Rearrangement. Angew. Chem. Int. Ed. 2010, 49 (50), 9734-9736. https://doi.org/10.1002/anie.201004778.

(49) Trost, B. M.; Yasukata, T. A Catalytic Asymmetric Wagner-Meerwein Shift. J. Am. Chem. Soc. 2001, 123 (8), 7162-7163. https://doi.org/10.1021/ja010504c.

(50) Wu, H.; Wang, Q.; Zhu, J. Organocatalytic Enantioselective Vinylogous Pinacol Rearrangement Enabled by Chiral lon Pairing. Angew. Chem. Int. Ed. 2016, 55, 15411-15414. https://doi.org/10.1002/anie.201609911.

(51) Gao, A. X.; Thomas, S. B.; Snyder, S. A. Pinacol and Semipinacol Rearrangements in Total Synthesis. In Molecular rearrangements in organic synthesis; Rojas, C. M., Ed.; Wiley, 2015; pp 2-33.

(52) Rojas, C. M. Molecular Rearrangements in Organic Synthesis; Wiley, 2015. https://doi.org/10.1002/9781118939901.

(53) Gergel, S.; Soler, J.; Klein, A.; Schülke, K. H.; Hauer, B.; Garcia-Borràs, M.; Hammer, S. C. Directed Evolution of a Ketone Synthase for Efficient and Highly Selective Functionalization of Internal Alkenes by Accessing Reactive Carbocation Intermediates. ChemRxiv 2022, January 5, (date accessed 2022-01-07). https://doi.org/10.26434/chemrxiv-2022-dp9. 